\title{
Tra fonti erudite e lettori ordinari: una traduzione seicentesca del Satyricon
}

\author{
CORINNA ONELLI \\ EHESS-CRH-Grihl, Parigi
}

\section{Descrizione e contenuti di Ang}

Questo articolo presenta i primi risultati della ricerca che sto conducendo intorno ad una traduzione inedita del Satyricon in italiano. Questo primo contributo si interesserà in particolare al rapporto che il testo italiano intrattiene con le proprie fonti. Il manoscritto che trasmette la traduzione è conservato presso la Biblioteca Angelica di Roma con segnatura 2061 ed è stato descritto da Vitale negli Inventari dei manoscritti delle Biblioteche d'Italia (Vitale 1934, 212). ${ }^{1}$ Il manoscritto (d'ora in poi, Ang) è cartaceo, misura $210 \times 137 \mathrm{~mm}$ e consta in tutto di 114 carte (sono bianche la prima e le ultime 3 carte). Vitale stima che Ang risalga alla fine del Seicento. Dal punto di vista materiale, Ang è un libro di fattura piuttosto povera, semplicemente rivestito in pergamena floscia. Sulla coperta del piatto posteriore, si intravvedono delle note manoscritte, riferibili a quantitativi di farina, orzo e fave. La traduzione è stata vergata da un'unica mano, particolarmente regolare e ordinata (vedi imm. 1 e 3). Fatta eccezione per un numero ristrettissimo di integrazioni e per la cancellatura di una singola parola, Ang non presenta correzioni. Come argomenterò in seguito, ritengo che Ang sia stato redatto da un copista professionista.

La traduzione è anonima e non vi è alcun elemento che consenta di risalire al traduttore o agli antichi possessori del manoscritto. La traduzione sembrerebbe del tutto ignota alla storiografia della letteratura italiana. La prima menzione che la riguarda, a mia conoscenza, data al 1876, anno in cui il filologo e lessicografo Pietro Fanfani (1815-1879) pubblica un articolo dedicato alle traduzioni in ita-

\footnotetext{
${ }^{1}$ Il manoscritto è registrato anche nel database digitale Manus, a cura dell'Istituto Centrale per il Catalogo Unico.
} 
liano del Satyricon. Nell'articolo, decisamente critico nei confronti dei coevi traduttori italiani, Fanfani descrive brevemente una traduzione manoscritta del Satyricon in suo possesso, che mette a confronto con i Successi di Eumolpione pubblicati nel 1678 e le traduzioni di Vincenzo Lancetti e Giambattista Cely Colajanni, rispettivamente del $1806-7$ e del $1871 .^{2}$

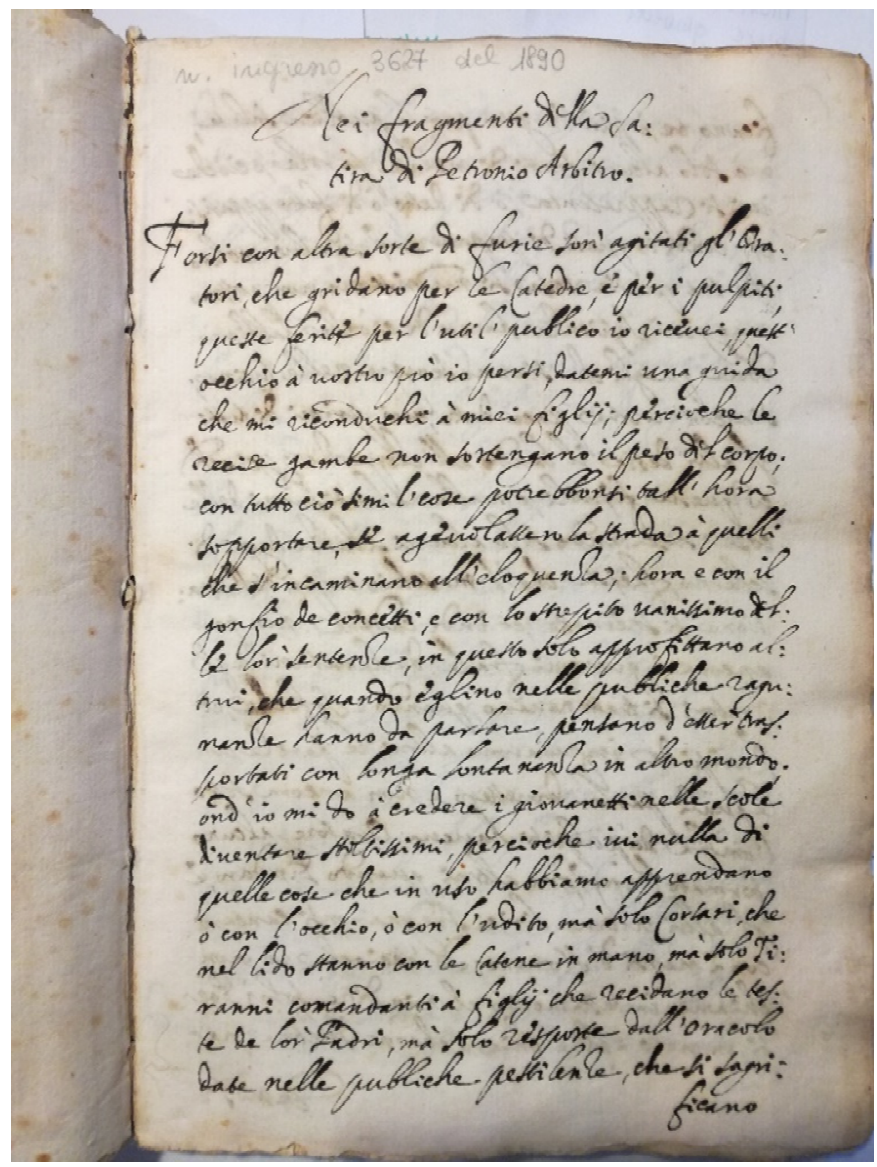

Imm. 1. Biblioteca Angelica, ms 2061 (Ang), c. $2^{\text {r }}$ (su concessione del Ministero per i Beni Culturali e del Turismo - Divieto di riproduzione). L'annotazione a matita sul margine superiore registra l'acquisto del manoscritto da parte della Biblioteca, avvenuto nel 1890.

${ }^{2}$ L'articolo di Fanfani, da contestualizzare nell'ambito dell'acceso dibattito sulla lingua italiana, è sintetizzato da Rini 1937, 88-89; da quest'ultimo ho appreso dell'esistenza della traduzione manoscritta. Fanfani fu tra i più noti esponenti del purismo linguistico dell'Ottocento, vedi Serianni 1990, 45-46 e 79-81. Per i Successi di Eumolpione, parziale traduzione del Satyricon, mi permetto di rinviare a Onelli 2012. 
Fanfani cita dunque un estratto della traduzione inedita (si tratta del passo corrispondente a Sat. 27), il cui testo coincide, al netto degli interventi normalizzanti del purista Fanfani, con il testo di Ang. Che quest'ultimo sia proprio il manoscritto un tempo posseduto da Fanfani è confermato ad abundantiam dal fatto che il catalogo topografico della Biblioteca Angelica registra Ang come ingresso proveniente dalla biblioteca privata di Fanfani, che fu collezionista e bibliofilo e i cui manoscritti vennero acquisiti nel 1890 dalla Biblioteca (Sciarra 2009, 278). Infine, nel manoscritto, proprio in margine della traduzione di Sat. 27, troviamo una annotazione di mano ottocentesca, con ogni probabilità dello stesso Fanfani (vedi imm. 2).

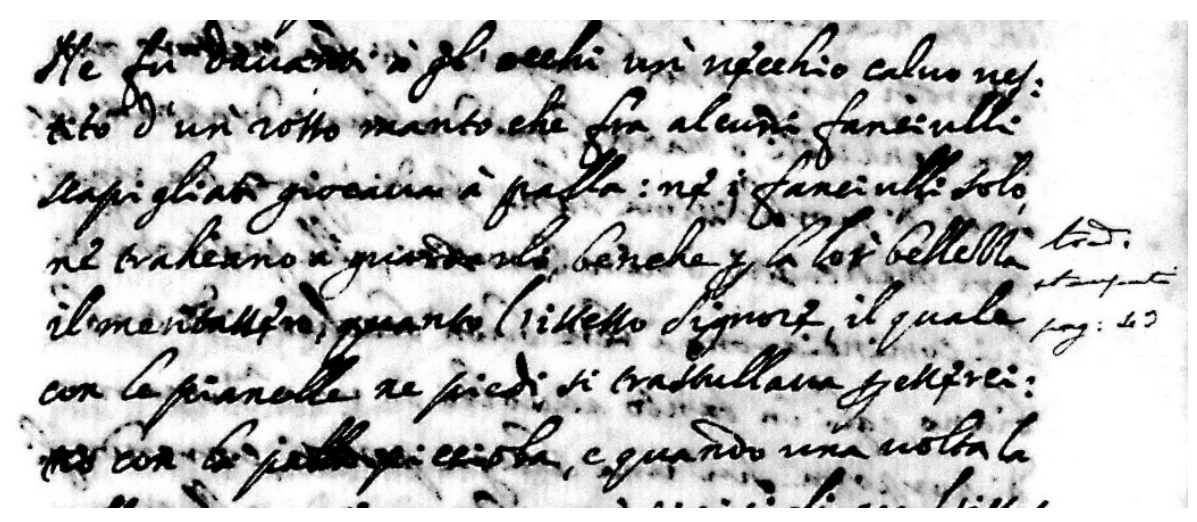

Imm 2. Biblioteca Angelica, ms 2061 (Ang), c. 17 $7^{\mathrm{r}}$, dettaglio (su concessione del Ministero per i Beni Culturali e del Turismo - Divieto di riproduzione). Nella nota a margine si legge: testo stampato pag: 4(?). Potrebbe trattarsi di un rimando di Pietro Fanfani ai Successi di Eumolpione (Napoli, 1678). Nel suo articolo Fanfani mette infatti a confronto la versione di Sat. 27 della traduzione manoscritta con quella pubblicata nei Successi di Eumolpione, che inizia a p. 42.

Ad un primo esame, la traduzione copiata in Ang sembrerebbe basarsi su un testo del Satyricon di tipo L, corrispondente cioè all'estensione degli excerpta longiora; quindi, grosso modo: Sat. 1-26,6; 27-37,5; 55; e 79-141 delle odierne edizioni che, ricordo, constano in tutto di 141 capitoli. La traduzione italiana omette dunque il testo tràdito dal frammento di Trau, ovvero il celeberrimo episodio della Cena Trimalchionis. ${ }^{3}$

Riporto la descrizione di Ang come effettuata da Vitale 1934 (traduco dal latino e sciolgo le sigle):

\footnotetext{
${ }^{3}$ Scoperto intorno al 1650, il frammento copre Sat. 26,7-78,8 sovrapponendosi quindi nella primissima parte al testo di estensione degli excerpta longiora. Pubblicata la prima volta nel 1664 come testo a sé stante, la Cena è stata incorporata nel testo del Satyricon a partire da Petronius 1669.
} 
1. T. Petronio Arbitro, Satyricon (tradotto in italiano da Giulio Cesare Becalli?), fol. 2-84a [...];

2. «Terenziano Mauro (?) sovra il iambico di doi metri detto il zoppo» fol. $84^{\mathrm{b}}[\ldots]$ segue un frammento che inizia con «Una vecchia ubbriaca»;

3. Dello stesso Petronio Arbitro, Frammenti del Satyricon, fol $86-89^{\mathrm{a}}[\ldots] ;^{5}$

4. Di incerto, Pervigilium Veneris tradotto in italiano in versi sciolti con il titolo «Scherzi lascivi», fol. 91 ${ }^{\mathrm{b}}-93^{\mathrm{b}}$;

5. «Raccolta di varie poesie de' Poeti» [...] Incipit «Bacco inventor delle viti», fol. 94-111.

Anzitutto, andrà notato come Vitale non trascriva nella sua scheda l'effettivo titolo della traduzione, che è - forse inaspettatamente - Nei fragmenti della Satira di Petronio Arbitro e non Satyricon (vedi imm. 1). Avrò modo di tornare su questo elemento, voglio ora piuttosto sottolineare il fatto che i componimenti segnalati da Vitale ai punti 2-4-5 come disorganici rispetto al Satyricon andranno invece considerati parte integrante del 'Satyricon' inteso come prodotto editoriale. I componimenti segnalati al punto 2 (Terentiano Mauro...Una vecchia ubbriaca...) sono infatti la traduzione in italiano di alcuni dei frammenti petroniani giunti per tradizione indiretta; ${ }^{6}$ mentre l'insieme costituito dalla traduzione del Pervigilium Veneris (punto 4) e la Raccolta di varie poesie (punto 5) altro non sono se non la versione in italiano della antologia intitolata Errores Venerii édita per la prima volta da Pierre Pithou (1539-1596) in appendice alla sua seconda edizione del Satyricon (= Petronius 1587). La stessa antologia verrà successivamente ripresa nelle seguenti edizioni: Petronius 1601, 1608, 1615, 1618a e, con lievi modifiche, in Petronius 1629a. Occorre tuttavia osservare che nell'edizione di Pithou, e nelle altre appena menzionate, gli Errores Venerii sono preceduti dalla raccolta Lusus ... in Priapum (= i Priapea), che invece non troviamo tradotta in Ang. ${ }^{7}$

${ }^{4}$ Vitale scrive 'Becalli', ma dovrebbe trattarsi di Giulio Cesare Becelli (1683-1750), al quale è in effetti attribuita una traduzione di Petronio (Rini 1937, 107-108). Tuttavia, come apparirà evidente nel prosieguo dell'articolo, ipotizzare che il purista Becelli possa essere l'autore della traduzione tràdita da Ang è del tutto incongruo, per ragioni cronologiche, linguistiche e stilistiche.

${ }^{5}$ Si tratta della traduzione dei Fragmenta così come éditi in Petronius 1587 e successivamente ripresi in numerose edizioni del Satyricon.

${ }^{6}$ Sono i seguenti frammenti, che cito seguendo la numerazione di Bücheler (= B.) e di Müller (= M.) impiegata rispettivamente in Petronius 1862 e 2009: fr. 19 B. e M. (da $2489 a t$ Arbiter disertus...); fr.21 B. e M.; fr.20 B. e M. (solo 61-65); l'incipit del fr.27 B. = fr. 28 M.; fr.26 B. =fr.44 M. 1-2; fr.25 B. e M.; fr.13 B. e M.; fr.16 B. e M.; $f r .15$ B. e M.; $f r .17$ B.; citazione da Giovanni di Salisbury (Policrat. 3,8: non duco contentionis...); fr.53 B.

${ }^{7}$ Gli Errores Venerii includono 89 componimenti di provenienza eterogenea, perlopiù di natura erotica, i quali costituiscono il nucleo della raccolta che verrà nel secolo successivo 
Un altro elemento al quale occorre prestare attenzione è il fatto che, come riportato da Vitale (punto 4), il Pervigilium Veneris, che è il primo dei componimenti degli Errores Venerii, si intitola nella traduzione italiana Scherzi lascivi. Dato che all'epoca il carme ancora non circolava sotto il titolo di 'Pervigilium Veneris ${ }^{8}$ e che nell' edizione di Pithou esso si trova di seguito al titolo della raccolta Errores Venerii, il traduttore ha probabilmente attribuito al solo poemetto e non all'intera raccolta il titolo Scherzi lascivi (che è chiaramente la traduzione di Errores Venerii), raggruppando i componimenti a seguire, da Floridi de qualitate vitae in poi (tradotto con Bacco inventor delle viti...cfr. punto 5 di Vitale), sotto il titolo Raccolta di varie poesie de' Poeti. Attribuisco invece ad una svista in fase di copiatura l'omissione del carme Ridiculum est cum te Cascam, diciannovesimo componimento degli Errores Venerii di Pithou. La Raccolta di varie poesie di Ang conta perciò un componimento in meno rispetto agli Errores Venerii, quindi 88 poesie in totale.

\section{Ang, un manoscritto clandestino}

Si può escludere categoricamente che Ang sia stato redatto dal traduttore in persona. Oltre all'estrema pulizia del testo, così come l'uso del richiamo (vedi imm. 3 ), che già di per sé potrebbero essere indicativi dell'opera di un copista professionista, sono soprattutto alcuni fraintendimenti presenti nel testo che mi spingono a concludere che Ang non sia stato scritto dal traduttore stesso.

Chi ha trascritto la traduzione ha infatti lavorato meccanicamente (probabilmente sotto dettatura), senza capire nomi e singole espressioni o talvolta orecchiando frettolosamente il senso del testo. Mi limito ad alcuni esempi: in Ang leggiamo Milone per il nome dello scultore Mirone, Teocle per Eteocle, da amaretta invece di da maretta, sinoltra per s'inoltra; o, ancora, in corrispondenza di Sat. 110,3 (agnovit Tryphena ... Gitona), abbiamo ricognobbe Trifena ... Trifone, in luogo di Gitone.

costituendosi come Anthologia Latina. L'insieme costituito dai Priapea e dagli Errores Venerii, si trova pubblicato, nelle edizioni ora menzionate, sotto al titolo Catalecta Veterum Poetarum.

${ }^{8}$ Allo stesso Pithou si deve il rinvenimento e l'editio princeps (1577) del Pervigilium Veneris, che il filologo francese conobbe mediante il codice Thuaneus. In questo testimone il poemetto è trascritto senza titolo. Solo successivamente verrà rinvenuto il codice Salmasianus (noto a partire dal 1615), nel quale il poemetto è introdotto dall'inscriptio 'Pervigilium Veneris', vedi Formicola in Pervigilium Veneris 1998, 20-21. 


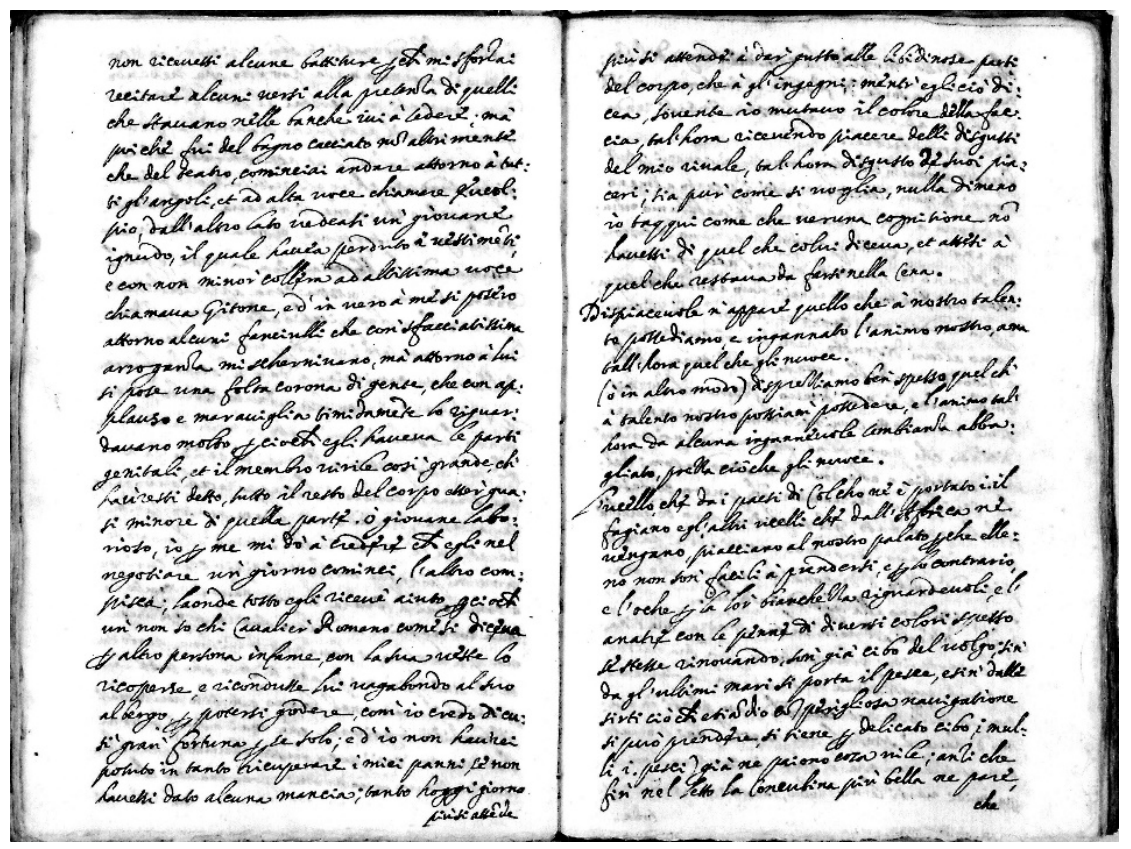

Imm. 3. Biblioteca Angelica, ms 2061 (Ang), cc. 34 $-35^{\mathrm{r}}$ (su concessione del Ministero per i Beni Culturali e del Turismo - Divieto di riproduzione). Il richiamo è presente in basso a destra, sia sul verso (più si attende) che sul recto (che).

Di particolare interesse è poi l'esempio che segue: in Sat. 119,33 Petronio descrive la fastosità dei banchetti nei quali viene servito lo scaro, pesce di lusso, che veniva condotto vivo a tavola ancora 'immerso nell'acqua del siculo mare' (trad. di Aragosti, in Petronius 1995). Ora, in Ang si legge, in corrispondenza di questo verso: sin da' Mari di Sicilia i tondi si traggano vivi. Quell'inspiegabile tondi a mio avviso doveva essere in origine tonni, tramutato in tondi dal copista. Come noto, l'assimilazione del nesso latino -ND- in -nn- (cfr. quanno, monno per quando, mondo) è tratto comune a buona parte dei dialetti dell'Italia centro-meridionale. Penso dunque che nell'intendere tonni il copista sia stato portato per ipercorrettismo a scrivere tondi per tonni. Un ulteriore esempio sembra confermare l'origine centro-meridionale del copista, nonché la sua dialettofonia di fondo: in corrispondenza della traduzione di Sat. 136,13 leggiamo infatti cusì gran grido arzò dove possiamo notare il rotacismo di [1] preconsonantico (arzò per alzò), fenomeno anche questo caratteristico dei dialetti centro-meridionali.

La traduzione copiata in Ang reca inoltre traccia di un successivo intervento di revisione. Abbiamo infatti un centinaio di glosse, interpolate nel testo continuo, sempre redatte dallo stesso copista, ma distinte dal testo principale tramite parentesi e da espressioni quali o vero, o, o in altro modo, i.(d est) (vedi imm. 4). 


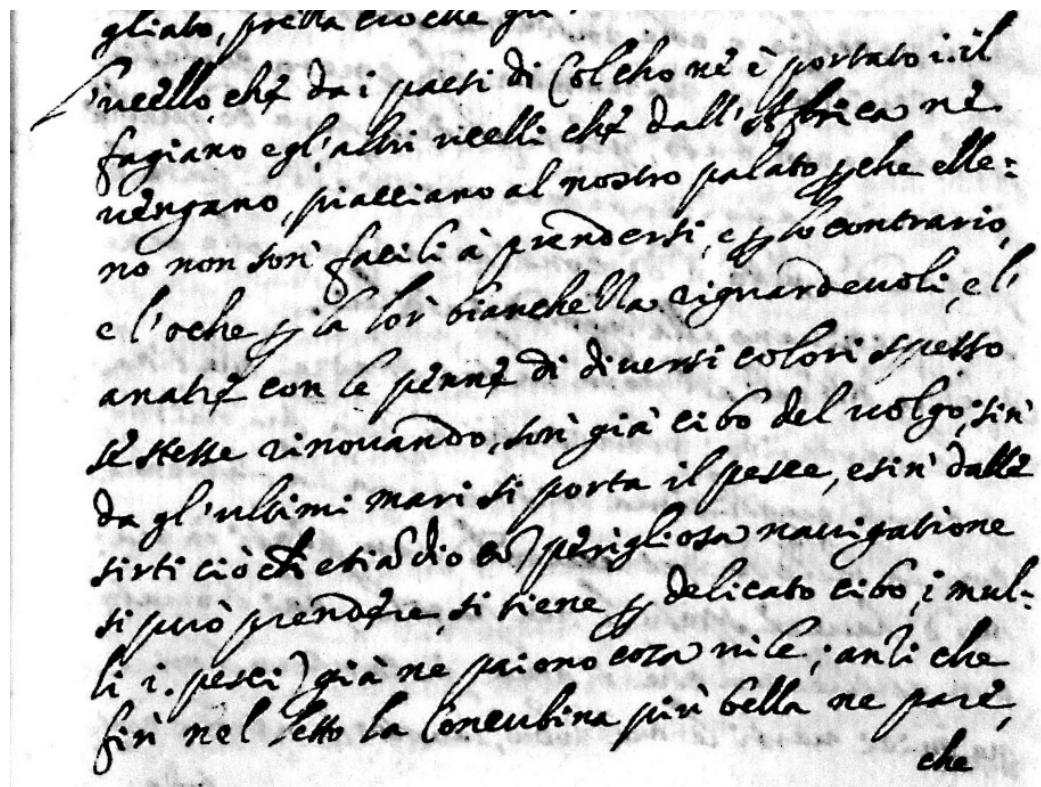

Imm. 4. Biblioteca Angelica, ms 2061 (Ang), c. 35 , dettaglio (su concessione del Ministero per i Beni Culturali e del Turismo - Divieto di riproduzione). Il passo corrisponde alla traduzione di Sat. 93, 2, 1-9. Si notino le due glosse interpolate: l'ucello che dai paesi di Colcho ne è portato $i$. il fagiano (prima riga) e si tiene per delicato cibo i mulli i. pesci) (penultima riga).

In base alla loro funzione, le glosse possono essere divise in due distinte tipologie: abbiamo glosse che fungono da commento esplicativo al testo del Satyricon; altre invece, e sono le più numerose, non commentano Petronio, ma parafrasano singoli termini della traduzione. Quello che colpisce è che in quest'ultimo tipo di glossa troviamo sistematicamente un italiano più moderno rispetto al termine impiegato nella traduzione. Così, ad esempio il termine pottaggio viene glossato con una vivanda assai magnifica; rimprocciarlo con sgridarlo, n'approveggi con n'approfitti; il vertadiero con il vero; origliero con guanciale; s'eregghi con s'innalzi. ${ }^{9}$

Certamente è da mettere in rilievo come nelle glosse, per rendere maggiormente comprensibile l'italiano della traduzione, si ricorra ad una varietà linguistica che non solo differisce dalla traduzione in termini diacronici ma anche dal punto di vista diastratico, nel senso che nelle glosse si possono leggere espressioni

\footnotetext{
${ }^{9}$ Nei casi appena elencati, la parafrasi è introdotta da $i$.(d est), tuttavia abbiamo casi di riformulazioni semplicemente giustapposte al termine parafrasato, p. es. in Ang si leggono raddoppiamenti come lo menò, lo trasse oppure col capo, con la faccia scoperta. Avverto del fatto che in questa sede ho optato per una trascrizione modernizzante del testo di Ang (mantengo tuttavia la $h$ etimologica, come in humido).
} 
di registro popolare o addirittura dialettali. Se infatti la lingua della traduzione non mostra particolari inflessioni regionali e cerca di mantenersi ad un livello letterario, nelle glosse troviamo espressioni decisamente basse, come in corrispondenza della novella della Matrona di Efeso dove l'elegante victor(que) miles utrumque persuasit $($ Sat. 112,2) viene ricalcato alla lettera nella traduzione con egli vincitore l'uno e l'altro ottenne (ovvero che la vedova accettasse dal soldato e il cibo e le avances). La glossa invece chiosa con un esplicito: $i$. che lei vivesse e li facesse servizio, termine quest'ultimo da intendere nell'accezione volgare, tuttora vitale, di 'prestazione sessuale'. Analogamente, l'espressione la sazietà dei cibi impiegata dal traduttore è parafrasata con un concretissimo: $i$. l'haver magnato e buto ben bene (si notino i participi passati magnato e buto, decisamente lontani dal registro letterario). Come espressioni fortemente connotate dal punto di vista geografico presenti nelle glosse segnalo: garognole per 'malleoli'; vaccio per 'rapidamente' e pancella per 'grembiule'. Sono tutte, queste, espressioni caratterizzanti i dialetti parlati tra Toscana (aretino) e Umbria settentrionale. ${ }^{10}$

Ora, a me pare del tutto improbabile che le glosse che esplicitano il testo italiano possano risalire al traduttore stesso, tanto più che chi ha commentato la traduzione talvolta ha frainteso il significato della stessa. Ad esempio, in Sat. 28,3, Petronio impiega il termine iatraliptae, che vuol dire 'medici-massaggiatori, fisioterapisti' (Schmeling 2011, 91), e che nella traduzione italiana è reso con ungitori medicastri. Ma la glossa che dovrebbe spiegare il significato di questa espressione in realtà ne dà una interpretazione erronea: ungitori medicastri ( $o$ vero, carnefici) - il fraintendimento potrebbe essersi originato dall'aver inteso ungitori nel senso degli 'untori' di manzoniana memoria. ${ }^{11}$

Escludo inoltre che possa essere stato il copista stesso a ideare il commento alla traduzione, dal momento che quest'ultimo rivela di non prestare attenzione né al senso del testo né tantomeno a quello delle glosse. Vediamo due esempi: quasi da poppa verginella scappò fuori dall'humido lembo (i. petto) e, in corrispondenza di Sat. 121, 111: veggio i campi de' doi Filippi (i. dove Cesare e Pompeo guerreggiavano). In entrambi gli esempi il copista ha mal posizionato le glosse esplicative: la prima, più congruamente, dovrebbe riferirsi a poppa e non a lembo; la seconda dovrebbe invece riferirsi alla battaglia di Farsalo, cui si fa allusione nel verso successivo (non sfugga inoltre che nel testo di Ang il nome della città Filippi è stato erroneamente interpretato - presumibilmente dal copista stesso

${ }^{10}$ Sui quali vedi Mattesini 1992, 509; Pellegrini 1982, 198. Non approfondisco in questa sede l'analisi dei tratti dialettali rilevabili nel testo di Ang.

${ }^{11}$ Erano detti untori coloro i quali erano ritenuti responsabili di diffondere intenzionalmente la peste tramite unguenti venefici. L'espressione viene comunemente associata alla peste di Milano del 1630 resa popolare dai Promessi sposi di Alessandro Manzoni (1785-1873). Tuttavia, la figura dell'untore è già ben documentata nel Cinquecento. 
- come plurale del nome di persona Filippo (de' doi Filippi). È quindi probabile che il copista avesse a disposizione un antigrafo che presentasse delle note esplicative a margine, dallo stesso interpolate nel testo continuo, talvolta senza attenzione.

Quanto finora esposto mi porta a concludere che Ang rappresenti la copia di un testo (la traduzione in italiano del Satyricon) che deve avere conosciuto una certa diffusione, in quanto riprodotto più volte, a distanza di tempo e addirittura commentato. Dato che il Satyricon era un testo proibito dall'Inquisizione - si noti che nessuna edizione di Petronio verrà data alle stampe in Italia dal 1499 al 1806 - ritengo che con ogni probabilità Ang rappresenti un manoscritto clandestino, prodotto professionalmente e destinato al mercato illegale. ${ }^{12}$

\section{La tradizione del Satyricon}

Se Ang è una copia, mi sembra opportuno chiedersi a quando possa invece risalire la traduzione. Distinguo quindi il testo materialmente trasmesso da Ang dalla traduzione, che chiamerò TR. Per stabilire a quando risalga TR, ho tentato di individuare l'edizione del Satyricon impiegata dal traduttore, compito peraltro facilitato dall'estrema letterarietà della traduzione, notata anche da Rini 1937, 89.

Il confronto sistematico di Ang con le edizioni seicentesche di Petronio ha rivelato un quadro a dir poco sorprendente, per descrivere il quale sarà necessario soffermarsi brevemente sulla tradizione del Satyricon. Escludendo il frammento di Trau (H), ${ }^{13}$ i testimoni del Satyricon si dividono in due classi: gli excerpta longiora $(\mathbf{L})$, alla quale ho già accennato, e gli excerpta vulgaria o brevia $(\mathbf{O})$, il cui testo copre grosso modo Sat. 1-26,5; 55; 80,9-137,9. Come si potrà notare, il testo trasmesso da $\mathbf{O}$ è interamente incluso nella classe $\mathbf{L}$. Questo vuol dire che il testo del Satyricon, se escludiamo $\mathbf{H}$, consta di parti $\mathbf{L O}$, trasmesse sia da $\mathbf{L}$ che da $\mathbf{O}$, e di parti $\mathbf{L}$, trasmesse esclusivamente dalla classe $\mathbf{L}$. Le due classi di estratti corrispondono a due diversi criteri di antologizzazione: i brevia sono contraddistinti

${ }^{12}$ Sebbene la condanna nei confronti del Satyricon emessa dal Concilio di Trento (15451553) appaia relativamente blanda, in quanto Petronio viene definito autore licenzioso da vietare ai fanciulli ma non agli adulti (Rini 1937, 41), occorre tenere presente che il decreto venne emesso quando ancora non si conoscevano gli excerpta longiora, che contengono i brani più scabrosi. Sulla circolazione manoscritta dei libri proibiti tra Sei e Settecento, si veda Barbierato 2012, 279-284 e relativa bibliografia.

${ }^{13}$ Non ritengo qui necessario accennare alla tradizione dei florilegi tratti dal Satyricon. Per informazioni più dettagliate sulla tradizione del Satyricon rinvio all'introduzione di Vannini in Petronius 2010, 39-61. 
dalla predilezione per le parti in poesia ed evitano accuratamente i brani a contenuto pederastico, trasmessi invece da $\mathbf{L}$.

La classe $\mathbf{O}$ è costituita da 3 importanti codici datati tra il IX e il XIII secolo e da una famiglia (ס) costituita da 15 manoscritti, tutti di epoca rinascimentale (XV sec.) e di provenienza italiana. L'autorità dei testi $\boldsymbol{\delta}$ è considerata pressoché nulla ai fini della costituzione del testo del Satyricon. Da un manoscritto $\boldsymbol{\delta}$ (oggi perduto) deriva l'editio princeps degli excerpta brevia, pubblicata a Milano nel 1482 ca. Peraltro, tale manoscritto doveva essere, nel novero della stessa classe $\boldsymbol{\delta}$, scadente, data la quasi totale omissione di Sat. 16-25,2 che contraddistingue la princeps e tutte le edizioni da essa derivate: la ristampa veneziana del 1499 e ancora l'edizione parigina del 1520. Tra i 15 manoscritti $\boldsymbol{\delta}$ a noi noti, quello maggiormente affine alle prime edizioni a stampa è il ms Barb. Lat. 4 (= Barb) della Biblioteca Apostolica Vaticana. ${ }^{14}$ Nel 1565 viene pubblicata ad Anversa quella che sarà l'ultima edizione basata sugli excerpta brevia: si tratta dell'edizione dell'umanista ungherese Sambucus (Janos Zsámboky, 1531-1584). Per la sua edizione, Sambucus si avvalse dell'edizione parigina del 1520 e di un manoscritto $\boldsymbol{\delta}$ in suo possesso che gli consentì di colmare le estese lacune in Sat. 16-25,2 presenti nelle edizioni precedenti (De la Mare 1976, 230). Oltre a questo evidente progresso, l'ed. Sambucus si contraddistingue per l'elegante veste umanistica e per il fatto di presentare a margine varianti, congetture e talvolta brevi note di commento.

Conosciamo gli excerpta longiora $(\mathbf{L})$ solo attraverso testimoni della seconda metà del Cinquecento, tutti derivati da due manoscritti medievali oggi perduti: il codice Benedictinus e il Cuiacianus. Intorno al 1571, Scaligero (Joseph Justus Scaliger, 1540-1609) ebbe a disposizione quest'ultimo, dal quale trasse una copia di proprio pugno (il manoscritto, oggi conservato presso la Universiteitsbibliotheek di Leida, è siglato I). Ma più che una semplice trascrizione del Cuiacianus, Scaligero produsse una vera e propria edizione di Petronio, mediante la conflazione di più fonti. Di più, il testo di $\mathbf{I}$ presenta certamente anche congetture dello stesso Scaligero.

La prima edizione a stampa degli excerpta longiora viene pubblicata a Lione nel 1575. Pur rappresentando 'una vera e propria editio princeps', la pubblicazione non rivendica la propria novità in alcun modo, rappresentando, come sottolinea Stagni, 'un modello di understatement più unico che raro per un tipografo del Cinquecento' (Stagni 1993, 227 n.50). L'edizione è detta tornesiana dal nome del tipografo Jean de Tournes (1539-1615) ed è siglata $\mathbf{t}$. Per le parti $\mathbf{L}$ da Sat. 1 a 112,4, il testo di $\mathbf{t}$ si fonda esclusivamente sul ramo derivante dal Benedictinus; in seguito, il testo di $\mathbf{t}$ è stato stabilito anche a partire dal Cuiacianus. Questo

${ }^{14}$ Rinvenuto negli anni Trenta del secolo scorso, Barb è descritto da De la Mare 1976, 225. 
perché gli editori di $\mathbf{t}$ ricevettero un exemplar vetustissimus (verosimilmente, il Cuiacianus) quando la stampa era ormai stata avviata. ${ }^{15} \mathbf{t}$ presenta inoltre un importante corredo di varianti a margine, per lo più di natura congetturale.

A due anni di distanza da $\mathbf{t}$, vede la luce la prima edizione di Pithou (= Petronius 1577), che all'epoca si poté avvalere, per le parti $\mathbf{L}$, solo del testo derivante dal ramo del Benedictinus. Successivamente, anche Pithou ebbe a disposizione il Cuiacianus, che impiegò per la sua seconda edizione (= Petronius 1587). Inoltre, rispetto alla prima, la seconda edizione di Pithou contempla: due commenti, ovvero le Notae e i Collectanea, entrambi anonimi ma attribuibili rispettivamente a Pierre Pithou e al fratello François (1543-1617) ${ }^{16}$ i Fragmenta, nonché, come già sottolineato, i Catalecta Veterum Poetarum accompagnati dalle relative Annotationes, da attribuire allo stesso Pierre Pithou.

\section{Una fonte pre-tornesiana}

Come anticipato, dalla ricerca dell'edizione verosimilmente impiegata dal traduttore italiano come testo di partenza è emerso qualcosa del tutto inaspettato e cioè che per le parti LO sino al lungo inserto poetico del Bellum civile (Sat. 119-124) il testo italiano non segue il testo $\mathbf{L}$, ma aderisce al testo di Petronio così come tramandato dagli excerpta brevia. In maniera più specifica, per questa porzione, che d'ora in poi indicherò come TR1 (= traduzione delle parti LO fino al Bellum Civile), il testo italiano si basa, innegabilmente, su testo affine al sottogruppo costituito da Barb, le prime edizioni a stampa (qui rappresentate dall'ed. parigina del 1520) e dall'ed. Sambucus. Il nesso fra TR1 e Barbcs è evidente, come si può evincere anche solo da un confronto parziale (evidenzio in grassetto le corrispondenze):

${ }^{15}$ Questa, la ricostruzione schematica. Per una disamina più articolata, vedi Stagni 1993, 118-223.

${ }^{16}$ Vannini in Petronius 2010, 54-55. Ufficialmente, entrambe le edizioni di Pithou sono anonime. Sulla reticenza di Pithou nel pubblicare gli excerpta longiora, cfr. quanto riportato da Richardson 1993, 11 e 14. 
Tab. 1. Confronto tra Ang, gli excerpta brevia (Barbes) e i longiora (L):

Barb $=$ Biblioteca Apostolica Vaticana, Barb. Lat. $4^{17}$

$\mathbf{c}=$ Petronius 1520

$\mathbf{s}=$ Petronius 1565 (ed. Sambucus)

$\mathbf{L}=$ Petronius $1587,1601,1608,1615,1618 \mathrm{a}$, e $1629 \mathrm{a}^{18}$

\begin{tabular}{|c|c|c|c|c|c|}
\hline Sat. & Ang & Barb & c & $\mathbf{s}$ & $\mathbf{L}$ \\
\hline 1,3 & sambuco & sansuco & sansuco & sampsucho & sesamo \\
\hline 4,3 & s'inaffiassero & irrigarentur & irrigarentur & irrigarentur & mitigarentur \\
\hline 4,4 & di Luciano & Lucianae & Lucianae & Lucianae & Lucilianae \\
\hline 6,1 & verso gl'horti & in hortis & in hortis & in hortis & motu \\
\hline 7,3 & fra certi vicoli & inter viculos & inter viculos & inter viculos & inter titulos \\
\hline 14,2 & comprar & emere & emere & emere & vendere \\
\hline 25,7 & $\begin{array}{l}\text { con quella } \\
\text { meretrice }\end{array}$ & in scorto & in scorto & in scorto & in secreto \\
\hline 81,1 & o Scolanio & aut Scolanius & aut Scolanius & aut Scolanius & antescholanus \\
\hline 88,5 & $o m$. & om. & $o m$ & om. & Lysippum \\
\hline 88,6 & iniquità & iniquitatis & iniquitatis & iniquitatis & antiquitas \\
\hline 88,9 & e dell'honesto & honestique & honestique & honestique & bonique \\
\hline 94,2 & Circlopio & $\begin{array}{l}\text { Ciclopius } \\
\text { (Circlopius in } \\
\text { margine) }\end{array}$ & Circlopius & Circlopius & Encolpius \\
\hline 95,1 & la serva & famula & famula & famula & fabula \\
\hline 95,9 & si difendea & defendebat & defendebat & defendebat & vindicabat \\
\hline 96,4 & om. & om. & om. & om. & $\begin{array}{l}\text { velut... } \\
\text { replebam }\end{array}$ \\
\hline 109,3 & Eumolpo & Eumolpon & Eumolpon & Eumolpon & Encolpion \\
\hline 109,7 & $\begin{array}{l}\text { con poco } \\
\text { pesante tratto }\end{array}$ & inani spira & inani spira & inani spira & spuma \\
\hline 111,4 & om. & $o m$. & $o m$. & $o m$. & $\begin{array}{l}\text { quotiens } \\
\text { defecerat }\end{array}$ \\
\hline 111,6 & $i v i$ & ibi & ibi & ibi & sibi \\
\hline 118,1 & $\begin{array}{l}\text { Molti giovani, } \\
\text { dicea Eumolpo }\end{array}$ & $\begin{array}{l}\text { Multos inquit } \\
\text { Eumolpus } \\
\text { iuvenes }\end{array}$ & $\begin{array}{l}\text { Multos inquit } \\
\text { Eumolpus } \\
\text { iuvenes }\end{array}$ & $\begin{array}{l}\text { Multos, inquit } \\
\text { Eumolpus, } \\
\text { iuvenes }\end{array}$ & $\begin{array}{l}\text { Multos, inquit } \\
\text { Eumolpus, ô } \\
\text { iuvenes, }\end{array}$ \\
\hline
\end{tabular}

${ }^{17}$ Ho consultato la copia digitale di Barb disponibile nella sezione 'Manoscritti digitalizzati' del sito della Biblioteca Vaticana, vedi $<$ https://digi.vatlib.it/view/MSS_Barb.lat.4 $>$.

${ }^{18}$ Impiego come testo-base dei longiora quello delle edizioni che presentano la raccolta $\mathrm{Er}$ rores Venerii. 
Nel sottogruppo rappresentato da Barbcs, TR1 sembrerebbe maggiormente affine a cs. Come questi ultimi, infatti, in corrispondenza di Sat. 26,1 Ang omette Psyche, mentre Barb ha $\psi v \chi \dot{\eta}$. Inoltre, in corrispondenza di Sat. 109,9 in Ang leggiamo per le spaziose campagne del cielo, che traduce il per inania di cs, quando Barb legge per maria.

Fra $\mathbf{c}$ e $\mathbf{s}$, TR1 sembrerebbe più prossima a quest'ultimo:

Tab. 2. Confronto tra Ang, l'ed. Sambucus e l'ed. parigina del 1520:

$\mathbf{s}=$ Petronius 1565 (ed. Sambucus)

$\mathbf{s}^{\mathbf{m}}=$ variante a margine di $\mathbf{s}$

$\mathbf{c}=$ Petronius 1520

\begin{tabular}{|llll|}
\hline Sat. & Ang & s & c \\
\hline 1,3 & ma solo tiranni & sed tyrannos & et tyrannos \\
2,4 & aveva...formato & dolaverat $\left(\mathbf{s}^{\mathbf{m}}\right)$ & deleverat \\
\hline 2,6 & percioché & nam $\left(\mathbf{s}^{\mathbf{m}}\right)$ & et iam \\
\hline 17,9 & ridiciate & referatis & om. \\
\hline $18,6,4$ & veglia & vigilat & iugulat \\
\hline $18,6,4$ & ricever morte & obire & abire \\
\hline 25,4 & in ira di Giunone & iratam & irritatam \\
\hline 26,5 & mi bagiava & osculis verberabat & oculis v. \\
\hline $55,6,16$ & di lino & linea & lunae \\
\hline 95,8 & i sbirri & lictores & coctores \\
\hline 95,8 & vivande & escis & aestis \\
\hline 111,4 & asciugava & commundabat & commendabat \\
\hline 112,6 & con la commodità & commode & commodet \\
\hline 118,1 & leggiadramente & teneriore $\left(\mathbf{s}^{\mathbf{m}}\right)$ & teneriorem \\
\hline
\end{tabular}

Nonostante questi significativi riscontri, non ritengo si possa identificare con assoluta certezza s come testo di partenza di TR1. La ragione è che, come vedremo, TR1 è stata emendata sulla scorta di commenti di epoca post-tornesiana, nei quali non è raro ritrovare lemmatizzate varianti da $\mathbf{s}$. Questo vuol dire che se in Ang, in corrispondenza di Sat. 55,6,16 leggiamo ammanto di lino, che è chiaramente la traduzione di nebula linea, congettura di Sambucus per il tràdito nebula lun(a)e, non possiamo concludere che necessariamente $\mathbf{s}$ sia stato il testo di partenza di TR1. Ammanto di lino potrebbe infatti rappresentare un emendamento subentrato quando TR1 era già stata ultimata. Peraltro, non si può del tutto escludere che TR1 fosse basata su una fonte manoscritta. Per prudenza, preferisco quindi indicare la fonte di TR1 genericamente come 'pre-tornesiana'. 
Per quanto riguarda la traduzione delle parti LO dal Bellum Civile in poi, esse mostrano di dipendere decisamente da un'edizione $\mathbf{L}$, non presentando nessuna delle lacune di $\boldsymbol{\delta}$ (come ad esempio l'omissione di Sat. 120,80) o l'ordine perturbato di Sat. 134-135. Si registrano, è vero, alcuni eventuali punti di contatto con s: in corrispondenza, ad esempio, di Sat. 131,6,6 dove Ang ha il silvestre rusignolo (cfr. s: silvester aedon) o di Sat. 135,8,15-17: Hecale...figlio di Batto (cfr. s: Hecale ...Battiadae). Ma molto probabilmente questi casi non rappresentano lezioni derivate direttamente da $\mathbf{s}$, per la stessa ragione segnalata poc'anzi. $\mathrm{Si}$ tratta infatti di varianti che hanno goduto di ampia fortuna e che sono correntemente lemmatizzate nei commenti seicenteschi, sulla base dei quali è stata verosimilmente riletta TR1 (cfr. infra Parte 7).

\section{Una traduzione anonima}

Da Sat. 1 a 118 la traduzione in italiano segue dunque una fonte pre-tornesiana per le parti $\mathbf{O}$ e, evidentemente, un testo $\mathbf{L}$ per le parti $\mathbf{L}$. L'effetto complessivo è a dir poco straniante, perché i due rami della tradizione risultano letteralmente giustapposti. Per meglio illustrare il risultato finale, mi soffermerò su un esempio in particolare. Si tratta dell'episodio (Sat. 16-26) in cui i tre protagonisti (Encolpio, Gitone e Ascilto) si ritrovano da Quartilla, presunta sacerdotessa di Priapo. Quest'ultima li coinvolge in una sorta di rituale orgiastico, nel corso del quale si decide di deflorare una ragazzina, facendola 'sposare' all'adolescente Gitone. Si procede quindi all'allestimento di una cerimonia che parodia il rito nuziale: la ragazzina viene velata con il flammeo e si forma un improbabile corteo di donne ubriache.

Ecco il testo di Sat. 26,1 - si tratta di un contesto $\mathbf{L O}$ - come trasmesso da $\mathbf{L}$ : iam Psyche puellae caput involverat flammeo, iam embasicoetas praeferebat facem ('già Psiche [ancilla di Quartilla] aveva avvolto col flammeo il capo della ragazzina, già l'embasiceta, alla testa del corteo, portava innanzi la fiaccola', trad. di Aragosti in Petronius 1995). Se non vi è accordo tra gli studiosi sul significato proprio di embasicoetas ('vaso da notte', 'coppa per bere'? vedi Schmeling 2011, 71), è invece certo che da Quartilla il termine è impiegato metaforicamente per designare il cinedo. In precedenza, infatti, la padrona di casa in persona aveva spiegato ad uno spiazzato Encolpio in quale senso fosse da intendere l'esotico termine, esclamando: 'Ma come, non avevi intuito che è il cinedo a chiamarsi embasiceta?' (trad. di Aragosti in Petronius 1995). Ora, questa frase appartiene a Sat. 24,2, ovvero a una porzione testuale $\mathbf{L}$, quindi sconosciuta a $\boldsymbol{\delta}$ e di conseguenza alle prime edizioni a stampa del Satyricon. Come intuibile, per chi avesse 
a disposizione il solo testo $\boldsymbol{\delta}$ non poteva risultare agevole l'interpretazione di embasicoetas in Sat. 26,1 che viene infatti analizzato, in Barb e in buona parte dei manoscritti $\boldsymbol{\delta}$, come embasico/embafico (a)etas. ${ }^{19}$ E così viene ripreso da $\mathbf{c}$ e da $\mathbf{s}$, che entrambi riportano: iam puellae caput involverat flammeo, iam embasico aetas praeferebat facem. Se ora ci rivolgiamo alla traduzione italiana, possiamo facilmente constatare come essa non derivi da $\mathbf{L}$ ma da un testo assimilabile a Barbes:

Era della putta già involto il capo e coperto da un velo di bambage e già l'età gli portava avanti la fiaccola.

Oltre all'omissione di Psiche, quello che salta agli occhi è la traduzione l'età gli portava ..., che riprende letteralmente l'incongruo aetas praeferebat facem. Quanto alla traduzione di bambage ('di bambagia, cotone'), si tratta, io credo, del maldestro tentativo di dare un senso all'inesistente embasico, che deve essere stato messo in qualche modo in relazione con bambax, -acis (lat. mediev. 'cotone, bambagia').

Ma perché qui, così come in altri casi (cfr. tab. 1), il traduttore avendo a disposizione un testo $\mathbf{L}$ non ha impiegato direttamente questo invece di un'obsoleta fonte pre-tornesiana? La mia ipotesi è che TR1 preesistesse a TR2, nel senso che la traduzione italiana è stata iniziata a partire dalla fonte pre-tornesiana per poi essere continuata, da Sat. 118 in poi, sulla scorta di un testo $\mathbf{L}$, dal quale deriva il testo dal Bellum Civile alla fine e con il quale devono essere state colmate a posteriori le parti L di Sat. 1-118.

Non sono per ora emersi elementi che possano far pensare che alle due fasi della traduzione, pre- e post-tornesiana, corrispondano due diversi traduttori. L'impressione è in definitiva che il traduttore abbia mutato il proprio testo di partenza in corso d'opera, non mostrando quindi la benché minima preoccupazione di ordine filologico, anche a scapito dell'intelligibilità del testo italiano. Egli ha infatti unito le due tradizioni in modo del tutto acritico, percependo il testo $\mathbf{L}$ letteralmente come longior rispetto alla fonte pre-tornesiana, ovvero semplicemente come 'più lungo', quando la differenza fra le due tradizioni è anche qualitativa. L'esempio dell'embasicoetas mostra bene la grossolanità con cui è stata condotta l'operazione, dal momento che il traduttore non si è neanche preoccupato di rileggere TR1, lasciando in Sat. 26,1 una frase pressoché priva di senso e lasciando anche, in corrispondenza di Sat. 94,2 l'incomprensibile Circlopio per Encolpio (cfr. tab.1). E non ha nemmeno ritenuto rilevante aggiornare il titolo della traduzione, che con ogni evidenza ancora rimanda alla fase pre-tornesiana. Né Barb

${ }^{19}$ Vedi le trascrizioni di Beck in Petronius 1863. 
né c né s si intitolano infatti Satyricon ma, rispettivamente: Petronii Arbitri Satyrici fragmenta quae extant, Petronii Arbitri... Satyrae fragmentum e Petronii Arbitri Satyrici Fragmenta.

Ho già avuto modo di osservare come in generale la traduzione sia piuttosto fedele, talvolta al limite della sudditanza. Il traduttore ha difatti tradotto pressoché verbatim, circostanza piuttosto sorprendente per l'epoca. Tuttavia, alla luce di quanto finora esposto, credo si possa escludere che la stretta aderenza della traduzione al testo latino risulti da preoccupazioni di ordine filologico. Mi sembra invece che, tutt'al contrario, la fedeltà della traduzione derivi dallo scarso spirito critico con il quale il traduttore affronta il testo latino. Due esempi saranno sufficienti per delucidare quello che intendo. Fuorviato dalla punteggiatura, ${ }^{20}$ il traduttore ha mantenuto degli infinitivi che in italiano è assolutamente necessario coniugare, pena la grammaticalità della frase. Si confronti:

(sciscitari coepit) cuius tam crudeles manus in hoc supplicium durassent. $\mathrm{Me}$ ruisse...contumeliam aliquam (Sat. 105,11. Corsivo mio).

con la traduzione di Aragosti:

(cominciò a chiedere) di chi fossero le mani tanto crudeli che avevano avuto l'ardire di infliggerci un tale spietato castigo. Anche se una punizione l'avevamo meritata... (corsivo mio).

\section{E infine Ang:}

(cominciò a chiedere) qual mano fosse stata tanto crudele che avesse potuto sopportare il darne tal martirio. Haver meritato alcun supplicio... (corsivo mio).

Lo stesso avviene in corripondenza di Sat. 117,6-7, dove la serie di infiniti (exisse, accessisse) viene lasciata intatta in italiano: ... percioché essersi partito quel buon vecchio dalla sua città... Essersi aggiunto a questo danno il naufragio.

Il traduttore conosce insomma il latino - è evidente - ma si tratta di una competenza non certo solida, ma che anzi vacilla davanti a costrutti anche solo minimamente marcati come quelli appena visti. Parimenti, il traduttore appare in difficoltà davanti a termini anche lievemente insoliti; in particolare, è palese la sua

${ }^{20}$ Si tratta di due contesti pressoché certamente derivati da un'edizione a stampa, più in particolare dall'ed. Wouweren dalla quale cito (vedi. infra Parte 6). 
difficoltà di fronte ai grecismi. Mi limito a qualche esempio: all'esordio del banchetto di Trimalcione (Sat. 31,3) alcuni schiavi si prodigano a togliere le pellicine intorno alle unghie (paronychia...tollentibus) dei piedi degli invitati sdraiati sui triclini; il traduttore, evidentemente non capendo il termine paronychia, cerca comunque di rendere la frase in italiano azzardando che gli schiavi alzavano i bacili; in Sat. 98,1 Gitone è nascosto sotto a un letto, così bene da toccare con il viso le cimici (ipsos scyniphes ore tangebat), ma il traduttore palesemente ignora il significato di scyniphes e tenta di cavarsela anche qui a senso, traducendo l'istesse coltri toccava col petto e con la bocca.

Ancora, in Sat. 91,1 Encolpio ritrova Gitone che se ne sta appoggiato a un muro cum linteis et strigilibus. Se molti commentatori spiegano che Gitone sta qui tenendo in mano degli asciugamani e degli strigili, in considerazione del fatto che il ragazzo si è da poco allontanato dalle terme (Sat. 97,2), il traduttore si mostra del tutto ignaro dei commenti e del rimando alle terme, intende strigilibus come striglie per cavalli ed è quindi portato a tradurre il tutto con un alquanto incongruo: veddi Gitone ...ch'haveva in mano e la streglia e $i$ pannamenti per acconciare cavalli. Infine, nei Fragmenta, troviamo Phoebea chelys (fr.26 B. = fr.44 M. 5), che altro non vuol dire se non 'tartaruga (cara a Febo)', erroneamente tradotto come ucello diletto a Febo. Possiamo allora immaginare che il traduttore, ignorando l'autentico significato di chelys, abbia tentato anche qui di aiutarsi con il contesto e che sia stato indotto a ritenere che chelys significhi 'uccello' perché dal verso successivo si evince chiaramente che il termine si riferisce ad un animale che depone le uova.

Ang ci trasmette insomma una traduzione che di certo non è attribuibile ad un letterato o a un umanista. Non si tratta di una traduzione d'autore, la definirei perciò anonima ma in senso forte, vale a dire che non solo ignoriamo il nome di chi l'abbia realizzata, ma anche che, molto probabilmente, il traduttore deve essere stato un anonimo professionista, il quale, alla stregua del copista, ha lavorato su commissione e la cui identità è perciò destinata a rimanere ignota. ${ }^{21}$

\section{La seconda fonte della traduzione}

Tornando alle fonti di TR, ci occuperemo ora di individuare la seconda fonte impiegata per comporre la traduzione italiana, ovvero l'edizione del Satyricon da cui sono state tratte le parti $\mathbf{L}$ da $S a t .1$ a 118 e da 118 in poi indistintamente tutto il testo, senza dimenticare i Fragmenta e gli Errores Venerii (chiamo questa seconda

${ }^{21}$ Sulla debole autorialità del traduttore, con particolare riferimento all'editoria a larga diffusione dell'epoca, vedi Carnelos 2012 e Cointre 2014, 1193-1199. 
porzione TR2). La presenza di quest'ultimi mi ha portata a concentrare l'attenzione sulle edizioni del Satyricon che contemplassero tale raccolta, che sono, ricordo: l'edizione di Pithou del 1587 e a seguire: Petronius 1601, le edizioni lionesi per i tipi di Paul Frellon (Petronius 1608, 1615, 1618a) e Petronius 1629a. Ora, le edizioni del 1615, 1618a e 1629a appena menzionate riproducono il testo del $S a$ tyricon dell'ed. Goldast (= Petronius 1610). Questo comporta che anche esse presentano l'interpolazione della novella del vetro infrangibile tra $S a t .55$ e $79,{ }^{22}$ del carme Somnia, quae mentes ( $f r .30$ B. $=f r .43$ M.) all'altezza di Sat. 104,3 e del carme Haec ait et ( $f r .40$ B. $=f r .37$ M.) in Sat. 137,3. Non essendo questi passi presenti nella traduzione italiana, ho ristretto l'analisi all'edizione di Pithou e alle edizioni Petronius 1601 e 1608, basate invece sul testo dell'ed. Wouweren (vedi Schmeling e Stuckey 1977, 52-53) che ancora non presenta tali interpolazioni.

Le differenze tra il testo stabilito da Pithou e quello di Wouweren non sono certo eclatanti e consistono principalmente in: Sat. 36,2, dove Pithou espunge scilicet in altero ferculo, presente invece nel testo di Wouweren (cfr. Ang: cioè un altro imbandimento); in Sat. 80,6 dove Pithou integra ego <qui $>$... putabam, mentre l'ed. Wouweren legge ego ... putabam (cfr. Ang: Io mi diedi a credere...); e in Sat. 82,1 dove l'ed. di Pithou ha ormai la lezione derivata dal Cuiacianus: largioribus cibis excito vires, ${ }^{23}$ mentre l'ed. Wouweren legge ancora, come l'ed. tornesiana: cibis excito vires (cfr. Ang: acciocché le forze non si perdessero ...presi alcun cibo). Per quanto fragili, queste evidenze mi hanno spinta ad approfondire l'ipotesi di un legame fra TR2 e l'ed. Wouweren.

Comunemente, si ritiene che il tedesco Johannes Wouweren (van der Wouweren, Woverius, a Wower, 1574-1612) abbia pubblicato per la prima volta la propria edizione del Satyricon nel 1594 (ma cfr. Appendice). L'ed. Wouweren, pur non apportando alcuna novità di rilievo per quanto attiene il testo di Petronio, ha goduto nei primi decenni del Seicento di grande fortuna, testimoniata dal numero di riedizioni e ristampe (almeno otto) che si susseguiranno fino al 1634. Come sottolineato da Schmeling e Stuckey, sarà proprio tramite l'ed. Wouweren, solitamente pubblicata in piccolo formato e a prezzo abbordabile, che il Satyricon conoscerà una diffusione più generalizzata (Schmeling e Stuckey 1977, 11). L'edizione del 1596, pubblicata a Leida ex officina Plantiniana, è dedicata da Wouweren a Scaligero e rappresenta la prima edizione del Satyricon ad essere corredata da un variegato apparato di commenti. Questo comprende: le Notae e i

${ }^{22}$ La novella viene raccontata nel contesto della Cena di Trimalcione e nelle odierne edizioni si legge in Sat. 51. La novella era tuttavia già nota per tradizione indiretta attraverso il Policraticus di Giovanni di Salisbury (m. 1180). Quest'ultimo ebbe infatti modo di conoscere tutte le parti del Satyricon a noi note.

23 'Mi rimetto in sesto con un pasto più che abbondante', trad. di Aragosti in Petronius 1995. 
Collectanea dei Pithou; una silloge anonima di Observationes, largamente debitrice nei confronti dei Praecidanea di Janus Dousa (van der Does, 1545-1604); ${ }^{24}$ lo Spicilegium di Dousa figlio (1571-1596), e infine le Animadversiones attribuite allo stesso Wouweren, che propongono, a ben vedere, non pochi emendamenti di Scaligero. L'edizione è inoltre contraddistinta da una nutrita appendice di varianti che accorpa in buona sostanza materiale dall'ed. Sambucus, dall'ed. tornesiana, dalla varietas lectionum delle edd. Pithou, nonché da $\mathbf{l}$, il manoscritto di pugno di Scaligero. Mi preme aggiungere che l'influenza di quest'ultimo sulle Animadversiones di Wouweren andrebbe forse rivalutata, anche alla luce del giudizio di Pierre Pithou, che di Scaligero fu intimo amico. Secondo Pithou, l'ed. Wouweren plagerebbe il lavoro di Scaligero su Petronio e lo stesso Scaligero reputava Wouweren un gran plagiaire. ${ }^{25}$

Nella serie delle ripubblicazioni dell'ed. Wouweren, ho concentrato l'attenzione sulle ristampe francesi del 1601 e del 1608, per l'ovvia ragione che si tratta delle uniche pubblicazioni che ibridano il testo del Satyricon dell'ed. Wouweren con i Catalecta (e relative Annotationes) ripresi dalla seconda ed. Pithou. L'edizione parigina del 1601 presenta la particolarità di costituire quella che si definisce un'edizione condivisa, ovvero un'edizione i cui costi e guadagni venivano divisi fra più stampatori-librari a ciascuno dei quali spettava un lotto di copie in proporzione (Riffaud 2011, 128). Nel caso del Satyricon l'accordo venne stipulato fra ben 10 stampatori-librari, ognuno dei quali appose il proprio indirizzo alle copie di competenza. Da qui l'impressione che si tratti di 10 edizioni distinte, mentre in realtà esse vanno considerate emissioni di una stessa edizione. ${ }^{26} \mathrm{~L}$ 'edizione lionese del 1608 appare nella sostanza identica alla parigina ed è interessante rilevare come entrambe le pubblicazioni siano state stampate prive di qualsiasi autorizzazione ufficiale, costituendo di fatto delle edizioni pirata dell'edizione olandese del 1596 (vedi imm. 5).

${ }^{24}$ La prima edizione dei Pro Satirico Arbitri...Praecidaneorum libri tres (= Praecidanea) risale al 1583; in seguito, il fortunato commento di Dousa è stato ripubblicato in numerose edizioni del Satyricon. Le Observationes contemplano inoltre congetture tratte dallo Spicilegiorum commentarius (1580) di Jan Palmerius (Palmier) e dai Suspicionum libri novem di Janus Gruterius (Gruytere 1560-1627).

${ }^{25}$ Scaliger 2012, 475. Cfr. anche Grafton 1993, 492-493.

${ }^{26}$ Cfr. Schmeling e Stuckey 1977, 12. Dietro l'edizione parigina del 1601 si cela un notevole intreccio familiare e commerciale, tutto incentrato sulla figura di Christophe Beys (15751647), nipote scapestrato del celebre Christophe Plantin (n. 1520?) di Anversa, sotto la cui egida era stata pubblicata nei Paesi Bassi l'ed. Wouweren; per ulteriori dettagli, si veda Schmeling e Stuckey 1977, 11-15. Colgo l'occasione per segnalare una edizione apud Hubertum Hunot, sempre pubblicata a Parigi nel 1601, della quale un esemplare è conservato presso la Bibliothèque Nationale de France. 


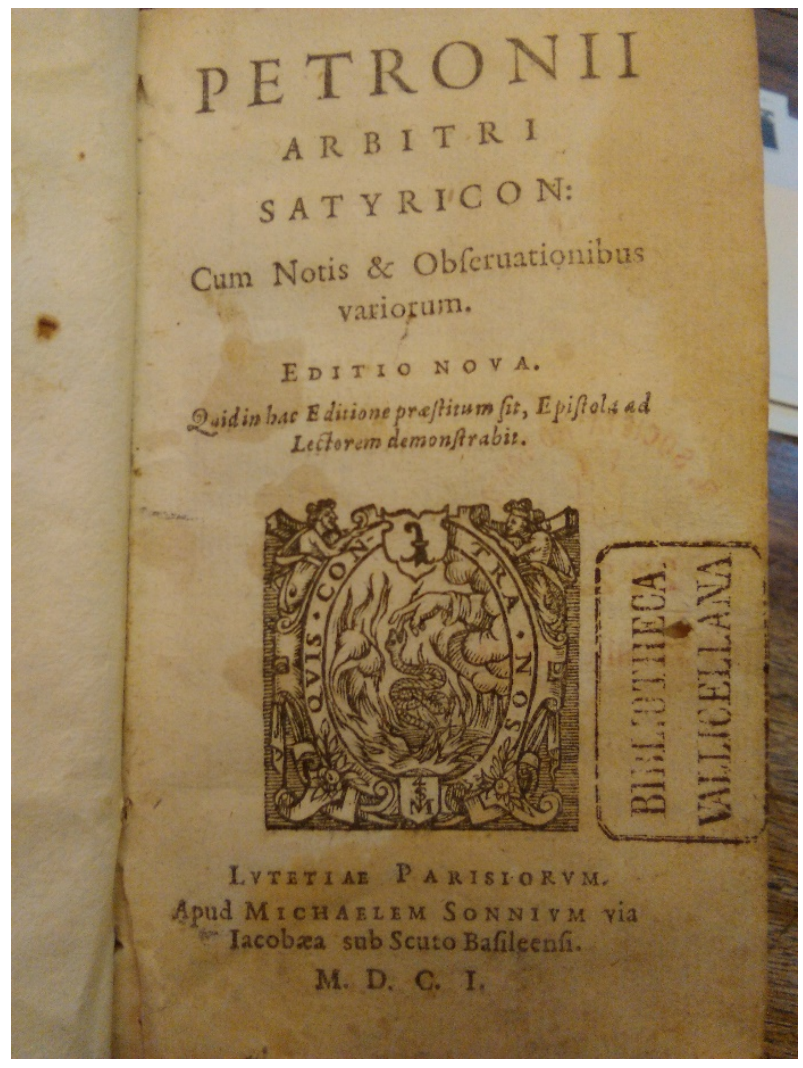

Imm. 5. Esemplare dell'edizione parigina del 1601 (in-12), emissione apud Michaelem Sonnium, frontespizio (Biblioteca Vallicelliana: S. BORR. P.V.116; su concessione del Ministero per i Beni Culturali e del Turismo - Divieto di riproduzione). Manca la menzione del privilegio, di norma apposta sotto la data, né all'interno del libro si trova alcuna autorizzazione alla pubblicazione.

C'è un dettaglio in particolare che sostanzia l'ipotesi di una dipendenza di TR2 dalle edizioni francesi. Quest'ultime si caratterizzano infatti per una scarsissima qualità tipografica, segno della frettolosità con cui è stata allestita la stampa. Tra i molteplici errori, ve n'è uno della massima importanza. In Sat. 124,251, il testo leggerebbe: Orbe fugax Ditis petit implacabile regnum. Le edizioni francesi ${ }^{27}$ hanno invece compattamente: Orbe sagax ...(vedi imm. 6).

${ }^{27}$ Oltre all'edizione lionese, ho potuto consultare tutte le emissioni dell'edizione parigina del 1601 elencate da Schmeling e Stuckey 1977, 52-53 ad esclusione dell'emissione apud Gulielmum Meleine. Anche l'emissione per i tipi di Hunot (vedi nota precedente) presenta il refuso, ma la escluderei come potenziale fonte di TR2 in quanto priva dei Catalecta. La 


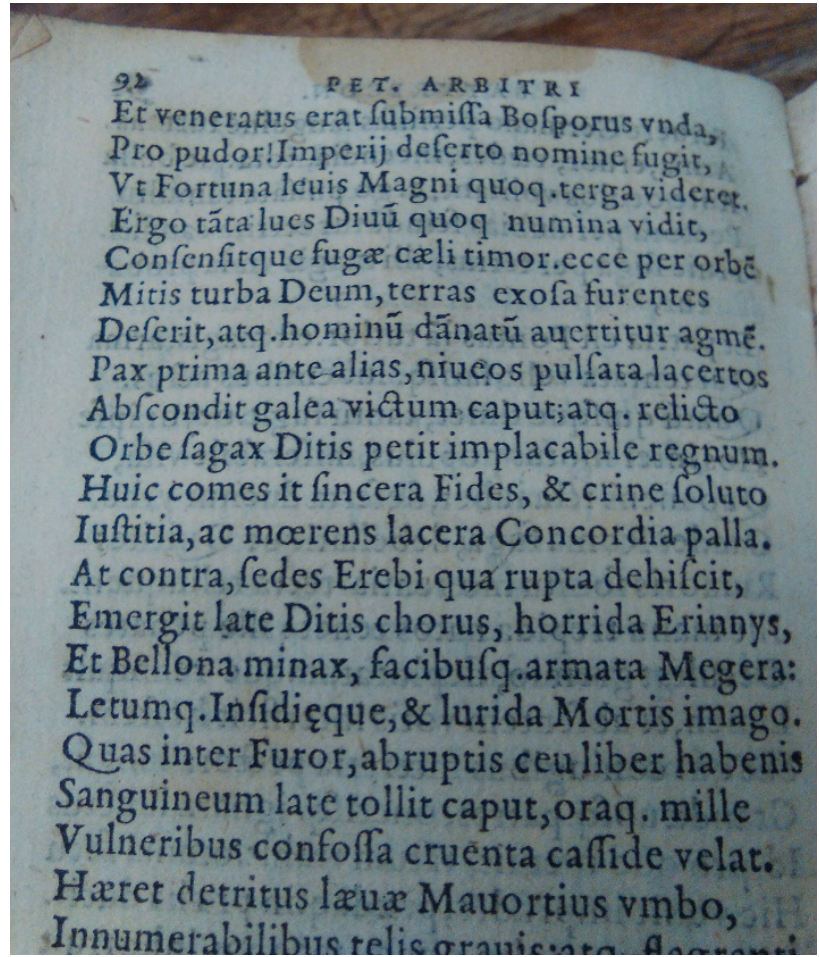

Imm. 6. Esemplare dell'edizione parigina del 1601 (in-12), emissione apud Michaelem Sonnium, p. 92, dettaglio (Biblioteca Vallicelliana: S. BORR. P.V. 116; su concessione del Ministero per i Beni Culturali e del Turismo). Alla r. 10, in corrispondenza di Sat. 124,251, si legge: Orbe sagax Ditis petit implacabile regnum.

Ora, in Ang la traduzione del verso è: la Pace ...sagace andonne all'implacabile regno dell'Inferno, che potrebbe spiegarsi appunto in quanto traduzione letterale dell' erroneo sagax per fugax. Lo stesso direi di Sat. 138,3, passo in corrispondenza del quale Ang ha senza paura fuggivo che potrebbe spiegarsi come traduzione del refuso secure fugientem per secutae, anch'esso riscontrabile nelle pubblicazioni francesi. Questo secondo caso appare tuttavia più complesso perché l'intera frase di Ang suona: per molti vicoli seguendo me che senza paura fuggivo. Il testo italiano sembra quindi contemplare al contempo la traduzione di secure ('senza paura') e di secutae ('seguendo'); sulla presenza in Ang di doppie lezioni, vedi infra Parte 9.

'lezione' sagax per fugax non si riscontra in nessun'altra pubblicazione dell'ed. Wouweren: Petronius 1596, 1604, 1614, 1618b, 1623, 1626, e 1634 (sul numero delle pubblicazioni dell'ed. Wouweren, vedi Appendice). 
Se, come ritengo, una delle ristampe francesi dell'ed. Wouweren è stata impiegata per produrre TR2, questo implicherebbe che il 1601 o il 1608 rappresentano il termine post quem per l'ultimazione della traduzione. Per quanto attiene il termine ante quem, ritengo che non ci si possa allontanare eccessivamente dal primo decennio del Seicento, dal momento che, come sappiamo, la traduzione è stata iniziata su una fonte pre-tornesiana e l'utilizzo di quest'ultima diventa sempre meno probabile con il trascorrere degli anni. Sembrerebbe dunque che Ang ci lasci intravvedere il primo esempio di volgarizzamento del Satyricon, primato finora comunemente attribuito alla fortunata traduzione in francese di François No$\operatorname{dot}($ c. $1650-1710) .^{28}$

\section{Varietas lectionum}

Ma, oltre i due testi-base che abbiamo individuato, la traduzione nel suo insieme sottintende l'impiego di ulteriori fonti. Non è infatti raro che, proprio in corrispondenza dei loci che ancora oggi attirano l'attenzione dei critici, la traduzione accolga emendamenti attribuibili a Wouweren, ai fratelli Pithou, a Dousa, a Scaligero, ma anche a fonti successive. Nel testo italiano si riconoscono infatti con nettezza non solo varianti e congetture eventualmente derivabili dall'apparato, identico, delle ristampe francesi dell'ed. Wouweren, ma, sorprendentemente, anche dall'ed. Goldast (= Petronius 1610), dall'ed. Gonzáles (= Petronius 1629b), nonché da ulteriori fonti, edite e inedite. Questi riscontri non possono non generare una serie di interrogativi, a partire dal numero di commenti che sarebbero stati consultati dal traduttore e dalla distanza temporale che sussiste tra la presunta data di ultimazione della traduzione e la pubblicazione dell'ed. Gonzáles. Ma soprattutto, a destare perplessità, è la profonda disparità fra la finezza del lavoro di critica che rivela il testo italiano in filigrana e il modus operandi del traduttore il quale, come abbiamo visto, ha piuttosto disinvoltamente cucito insieme testo pree post-tornesiano, per tacere delle sviste in cui è incorso nel tradurre.

Per affrontare questo quadro indubbiamente complesso, esemplificherò dapprima la presenza nella traduzione italiana di varianti eventualmente derivabili dall' apparato delle ripubblicazioni francesi dell'ed. Wouweren. Nella colonna 'variante' indico appunto la variante presumibilmente sottesa dalla traduzione

${ }^{28}$ Pubblicata la prima volta a Colonia nel 1693-1694 per i tipi di Peter Groth. Sulla traduzione-falso di Nodot, vedi Laes 1988. Segnalo tuttavia l'esistenza di una precedente traduzione in francese, anonima e ben più rara di quella di Nodot, pubblicata nel 1687 a Colonia con il titolo Pétrone. Traduction nouvelle (cfr. Schmeling e Stuckey 1977, 98). 
italiana, indicandone le fonti. Nella tabella riporto solo esempi da TR2 e utilizzo l'ed. Wouweren come testo-base (sottolineo le corrispondenze).

Tab. 3. Lezione e varianti da Petronius 1601 e 1608 a confronto con Ang:

Notae $=$ Notae di Pierre Pithou

Coll $=$ Collectanea di François Pithou

Observ. $=$ Observationes anonime

Anim. $=$ Animadversiones di Wouweren

$\mathbf{w}^{\mathbf{v}}=$ varietas lectionum di Wouweren

$\mathbf{t}=$ ed. tornesiana (Petronius 1575)

$\mathbf{t}^{\mathbf{m}}=$ variante a margine di $\mathbf{t}$

$\mathbf{I}^{\mathbf{m}}=$ variante a margine di $\mathbf{l}$, il manoscritto scaligeriano

$\mathbf{p}^{\mathbf{v}}=$ varietas lectionum delle edd. Pithou

\begin{tabular}{|c|c|c|c|}
\hline Sat. & ed. Wouweren & variante & Ang \\
\hline 9,2 & expressit & $\frac{\text { extersit }}{\text { (Fr. Pithou in Coll.) }}$ & cominciò ad asciuttarsi \\
\hline 12,2 & splendida vestis & $\frac{\text { splendor vestis }}{\left(\mathrm{da} \mathbf{I}^{\mathbf{m}} \text { in } \mathbf{w}^{\mathbf{v}}\right)}$ & $\begin{array}{l}\text { la vaghezza } \\
\text { cappa) }\end{array}$ \\
\hline 22,1 & sopitionibus & $\frac{\text { sopitis titionibus }}{\text { (Gruterius in Observ.) }}$ & $\underline{\text { con smorzati carboni }}$ \\
\hline 35,6 & mulos & $\frac{\text { mullos }}{\text { (Scaligero in Anim.) }}$ & sturioni \\
\hline 79,3 & scirpos & $\frac{\text { scrupos }}{\text { (Dousa in Observ.) }}$ & $\underline{\text { scogli }}$ \\
\hline 99,5 & $\begin{array}{l}\text { tamquam propudium } \\
\text { ignores }\end{array}$ & $\begin{array}{l}\text { prope diem, quia significat non longe } \\
\text { abesse diem (P. Pithou in Notae) }\end{array}$ & $\begin{array}{l}\text { quasi non sappi esser vicino } \\
\text { il giorno }\end{array}$ \\
\hline 100,4 & exciperet & $\frac{\text { exciperem }}{\left(\text { da } \mathbf{t}^{\mathrm{m}} \text { in } \mathbf{w}^{\mathrm{v}}\right)}$ & $\begin{array}{l}\text { lo vorrei dal suo essiglio } \\
\text { raccogliere }\end{array}$ \\
\hline 102,10 & mea & $\frac{\text { mihi }}{(\text { da } t}$ in $\left.\mathbf{w}^{v}\right)$ & $\underline{\text { mi tornò bene }}$ \\
\hline 103,6 & non & $\frac{\underline{\text { nos }}}{\text { (Dousa in Observ.) }}$ & $\underline{\text { noi }}$ \\
\hline 107,15 & $\begin{array}{l}\text { quae sola } \\
\text { salamandra }\end{array}$ & $\frac{\text { sola videtur inducendum }}{\text { (P. Pithou in Notae) }}$ & qual salamandra \\
\hline 107,15 & excussit & $\frac{\text { exussit }}{\text { (Dousa in Observ.) }}$ & $\underline{\text { ha...abbrugiato }}$ \\
\hline 114,4 & $\begin{array}{l}\text { postquam * manifesta } \\
\text { convaluit }\end{array}$ & $\begin{array}{l}\text { Postquam tempestas convaluit } \\
\text { (Fr. Pithou in Coll.) }\end{array}$ & $\begin{array}{l}\text { Doppo che la forza del vento } \\
\text { più fieramente si raddoppiò }\end{array}$ \\
\hline 134,2 & extricasti & $\frac{\text { excitasti }}{\text { (Wouweren in Anim.) }}$ & festi...adirare \\
\hline $136,6,2$ & pene- & $\frac{\text { sanie- }}{\mathbf{p}^{\mathbf{v}} \text { in } \mathbf{w}^{\mathrm{v} 29}}$ & feccie \\
\hline
\end{tabular}

${ }^{29}$ La congettura, attribuita a Pierre Daniel (1530-1603), si trovava già nella varietas lectionum delle edd. Pithou, che di Daniel fu amico (Vannini in Petronius 2010, 40). Le Notae di Daniel verranno pubblicate solo nel 1610, nell'apparato dell'ed. Goldast. 
Un analogo lavoro critico si riconosce nella traduzione dei Fragmenta, dove, ad esempio, il tràdito toto ...mero in $f r .33 \mathrm{~B} .=f r .29$ M. 2 è tradotto con l'istesso vino, che mi sembra riprendere il noto...mero proposto da Palmerius (ripreso in Observ.) e comunemente accettato dagli editori. Un altro caso rappresentativo si trova in corrispondenza di fr. $39 \mathrm{~B} .=f r .35 \mathrm{M} .4$ dove leggiamo né cessa il freddo horrore scorrere per l'ossa. Pithou aveva editato erigidus ... horror ('rigido orrore'), mentre, come si vede, la traduzione italiana recupera il tràdito (et) frigi$d u s . .$, reperibile in $\mathbf{w}^{\mathbf{v}}$.

Per gli Errores Venerii, mi limito qui all'esempio del Pervigilium Veneris, la cui traduzione in Ang rivela l'accoglimento della maggior parte degli emendamenti pubblicati da Pithou nelle sue Annotationes. In corrispondenza di PV 14, la traduzione italiana ha infatti fiato di Zefiro, cfr. con l'emendamento Favonii spiritum proposta da Pithou in luogo del tràdito faboni paritu; in corrispondenza di PV 32 leggiamo nudo (Pithou: nudus per durus); di PV 35 armato (Pithou: in armis per inermis); di PV $56^{30}$ al nudo (Pithou: nudo per nullo). Sempre il commento di Pithou, che riprende qui l'interpretazione di Giusto Lipsio (Justus Lipsius, Joost Lips, 1547-1606), spiegherebbe la traduzione di PV 73-74 che leggiamo in Ang: ...i Posteri di Romolo, onde e Giulio nascette e Cesare il nepote, dove il testo tràdito legge invece... posterum. / Romuli matrem crearet et nepotem Caesarem. Il soggetto di crearet è Venere, esaltata nei versi precedenti in quanto progenitrice della gens Iulia, con l'evocazione di Enea, di Romolo, per arrivare fino ad Augusto (nepotem Caesarem). Lipsio suggeriva di interpungere posterum Romuli, rendendo quindi il genitivo Romuli dipendente non da matrem ('madre di Romolo'), ma da posterum ('i posteri di Romolo') e, inoltre, di sostituire matrem con patrem. Secondo Lipsio, infatti, non sarebbe sensato trovare la madre di Romolo come penultimo termine nella sequenza celebrativa della gens Iulia. Da qui la necessità di leggere patrem ...et nepotem Cesarem, ovvero, rispettivamente, Giulio Cesare e Augusto. Come si sarà notato, la traduzione (e Giulio nascette...) aderisce in pieno all'interpretazione di Lipsio. ${ }^{31}$

Se ora ci rivolgiamo a TR1, possiamo notare come anche questa porzione testuale sia stata sottoposta ad un analogo procedimento di revisione, con ogni probabilità quando già era stata ultimata, dal momento che gli emendamenti che

${ }^{30}$ L'edizione di Pithou, ancora basata sul codice Thuaneus (vedi n.8), omette $P V 40$. Questo comporta uno sfasamento nella numerazione dei versi fra l'edizione di Pithou e le odierne edizioni. Nell'indicare i versi del Pervigilium Veneris, seguo la numerazione moderna.

${ }^{31}$ Il commento di Lipsio al Pervigilium Veneris è pubblicato nel suo Electorum liber I del 1580. Si può leggere anche nel terzo volume (Notae, castigationes, emendationes...) di Petronius 1629c, 266-270. Lipsio suggeriva inoltre di leggere in PV 81 explicant agni per explicat aonii. La traduzione italiana ( $\mathrm{gl}$ 'agnelli veggonsi) accoglie anche questo emendamento di Lipsio, che però non è riportato nelle Annotationes di Pithou. 
si riconoscono sono incontestabilmente di epoca post-tornesiana. Ad esempio: in corrispondenza di Sat. 2,7, in Ang leggiamo e insieme, che traduce il simulque proposto in primis da Wouweren per il semelque della classe $\mathbf{L}$ mentre Barbes hanno simulatq(ue); ${ }^{32}$ in corrispondenza di Sat. 6,3 si legge né in qual luogo fosse l'habitatione, traduzione che presuppone la soluzione [quia] nec <quo $>$ loco stabulum erat proposta sempre da Wouweren sulla scia di Dousa; in Sat. 55,6,2; in Sat. 84,1 la traduzione van dietro la traccia dell'honesta vita sottintende, in luogo del tràdito inspicere ('osservare'), insistere ('seguire') suggerito già da Wouweren; ${ }^{33}$ in Sat. 109,9,1 Ang legge quella parte che della bellezza è la maggiore che traduce la proposta di Dousa, ripresa nelle Observ., di sostituire quod solum formae decus con ...summum... mentre in Sat. 109,9,4 ha piange, traduzione che sottende necessariamente la congettura luget per ridet pubblicata dapprima in $\mathbf{t}^{\mathbf{m}}$ e poi in $\mathbf{w}^{\mathbf{v}}$.

Se gli emendamenti finora elencati sono virtualmente ricavabili dall'apparato di Petronius 1601 e 1608, come ho già anticipato, in Ang si riconoscono anche congetture apparse la prima volta nelle Symbolae di Melchior Goldast (15781635), pubblicate in appendice a Petronius $1610 .{ }^{34}$ Segnalo i seguenti riscontri: in corrispondenza di Sat. 107,9, commentando gratiam a legato, Goldast specifica 'legatum, id est a te' e in Ang leggiamo per tuo mezzo; in Sat. 108,10 in luogo di se abscissurum 'avrebbe squarciato' Goldast propone se abscisurum 'avrebbe tagliato, reciso' (cfr. Ang: avrebbe tagliato) e infine in Sat. 114,9, dove Goldast riporta il suggerimento di Jungermann di leggere da oscula 'dammi baci, baciami' (cfr. Ang: baciami) invece del tràdito ad oscula.

Anche dalla traduzione dei Fragmenta sembrerebbe che osservazioni riferibili al commento di Goldast abbiano guidato il traduttore: ad esempio, la traduzione di quae iacuere (lett. 'le cose che giacevano') di fr.36 B. =fr.32 M. 2 con quelle cose che pria erano sprezzate che presuppone un'interpretazione del tutto analoga

${ }^{32}$ Bücheler faceva invece risalire la congettura al commento di Goldast (Petronius 1862 in apparatu).

${ }^{33}$ La congettura insistere è oggi attribuita a Johann Alexander Brassicanus (c. 1500-1539), le cui note sono state pubblicate la prima volta in Petronius 1629c. Tuttavia, la stessa congettura si poteva già leggere, senza attribuzioni di sorta, nelle Animadversiones di Wouweren, quindi già dal 1596 .

${ }^{34}$ In realtà, le Symbolae sono pubblicate come opera di tale Georgius Erhardius, ma sono ormai comunemente attribuite allo stesso Goldast. L'imponente apparato dell'ed. Goldast è costituito da più di 20 commenti, tra i quali tutti e sei i commenti pubblicati da Wouweren nella sua edizione del 1596. Da segnalare che le Notae di Pithou sono però pubblicate da Goldast sotto la responsabilità di Christophe Richard. Inoltre, secondo Stagni, le note attribuite da Goldast a François Daniel, sarebbero anche esse di Pierre Pithou (Stagni cit. in Petronius 2010, 49). Nell'apparato dell'ed. Goldast si registrano anche due sillogi di note anonime. 
a quella di Goldast, secondo il quale iacuere è da intendere nel senso di 'essere negletti'; 35 così come la traduzione disfà di fr.49 B. 6 presuppone l'accoglimento della proposta di Pontanus, riportata da Goldast, di leggere teret ('distrugge') per il tràdito tenet.

Nel testo italiano si riconoscono inoltre emendamenti o integrazioni rapportabili al commento dell'ed. Gonzáles (= Petronius 1629b). Oltre al commento dello stesso José Antonio Gonzáles de Salas (1588-1654), l'edizione include in appendice (447-462) i Symbola di Caspar Schoppe (Schoppius, 1576-1649), redatti nel 1604. Valutiamo rapidamente alcune delle convergenze che mi sembrano sussistere fra la traduzione italiana e l'ed. Gonzáles:

Tab. 4. Confronto tra l'ed. Gonzáles e Ang

Gonz $=$ commento di Gonzáles

Schop $=$ commento di Schoppe

\begin{tabular}{|c|c|c|}
\hline Sat. & ed. Gonzáles & Ang \\
\hline 17,4 & $\begin{array}{l}\text { antecessura latrocinia... ego verior } \\
\text { puto: arte cessura..., id est artificiose } \\
\text { (Schop) }\end{array}$ & ingannevoli latrocinii \\
\hline 24,2 & $\begin{array}{l}\text { urbanitas vernulae fontem, placet } \\
\text { vernaculae (Schop) }\end{array}$ & fonte d'una civile piacevolezza \\
\hline 83,3 & honorabat, id est coronabat (Gonz) & del fiore nato da lui coronando \\
\hline 101,2 & $\begin{array}{l}\text { mors venit, quae nisi per te: malim } \\
\text { quae, si per te licet, id est si tuis } \\
\text { manibus moriamur (Schop) }\end{array}$ & $\begin{array}{l}\text { danne tu quella (la morte) con la tua } \\
\text { mano }\end{array}$ \\
\hline 114,2 & $\begin{array}{l}\text { Ego Gitoni adplicatus cum clamore } \\
\text { flevi (in textu) }\end{array}$ & Io abbracciato coi gridi Gitone dicevo ${ }^{36}$ \\
\hline 128,7 & socratica fide, hoc est honesta (Gonz) & d'amor honesto \\
\hline 129,10 & Lunam deducunt carminibus (Gonz) & $\begin{array}{l}\text { le donne co' loro incanti tolgono sin il } \\
\text { lume alla luna }\end{array}$ \\
\hline 136,2 & $\begin{array}{l}\text { Faciemque totam excitato cinere } \\
\text { perfundit: aqua scilicet effusa (Gonz) }\end{array}$ & $\begin{array}{l}\text { la faccia tutta imbrattossi con la cenere } \\
\text { che da quell'aqqua spruzzogli }\end{array}$ \\
\hline
\end{tabular}

35 Cfr. Sommariva 2010, 81.

${ }^{36} \mathrm{Si}$ potrebbe certo obiettare che: io ...Gitone, dicevo non è la traduzione di Ego Gitoni adplicatus. Ma potrebbe trattarsi di un errore del traduttore che avrebbe interpretato Gitoni come un vocativo, né si può escludere che l'integrazione presente in Ang non derivi dall'ed. Gonzáles ma dal manoscritto scaligeriano l, dove in corrispondenza di Sat. 114,2 si legge Ego Git. om . (ricavo il dato da Petronius 1863). Sulla presenza di fonti inedite nel testo di Ang vedi infra Parte 8. 


\section{Ulteriori fonti}

Ma le fonti riconoscibili in TR non si arrestano qui. Dall'analisi della traduzione degli Errores Venerii si evince infatti una certa familiarità con il commento di Scaligero in Vergilius 1573. Buona parte dei carmi éditi da Pithou in appendice al Satyricon erano infatti già stati pubblicati da Scaligero nella sua edizione dell'Appendix Vergiliana e Pithou, nelle proprie annotazioni ai Catalecta, riprende non pochi passi dal commento dell'amico Scaligero. Tuttavia in Ang si riconoscono alcune congetture di Scaligero che non sono riportate nelle Annotationes di Pithou e che perciò derivano necessariamente o dalla lettura del commento di Scaligero o da una fonte a questo ispirata. Ad esempio, in corrispondenza di [Verg.] Catal. 7,2 Ang ha mi ha hauto a ruinare questo putto, che traduce non il tràdito me perdidit iste potus ('questa bevuta mi ha fatto perdere la testa') dell'edizione di Pithou ma la congettura di Scaligero...putus ('questo ragazzo mi ha fatto perdere la testa'), congettura non riportata nelle Annotationes di Pithou. ${ }^{37}$

Ancora, in Ang in corrispondenza di Sat. 118,6 si legge: nel torrente dell'eloquenza, traduzione nella quale si riconosce l'accoglimento della congettura torrentem per il tràdito sententiarum tormentum. Il primo commento a segnalare questa congettura è quello di Pieter Burman (1669-1741) in Petronius 1709, che la derivava a sua volta dal commento di Caspar von Barth (1587-1658) a Stazio e da un passo non meglio identificato di Giusto Lipsio. Parimenti, la traduzione di Sat. 141,9: i cittadini di Sagunto... assediati sembra accogliere l'emendamento absessi per opressi riportato per primo sempre da Burman e da questi reperito nel commento di Konrad Rittershausen (1560-1613) a Oppiano. Escludendo l'ipotesi che TR sia stata corretta impiegando il commento di Burman, la presenza di queste due congetture in TR sembra rivelare una conoscenza certo non banale della letteratura esegetica in quanto si tratterebbe di una conoscenza estesa anche ad osservazioni sul testo petroniano disseminate in commenti su altri autori (cfr. n. 31).

Altri indizi rintracciabili in TR portano addirittura a pensare alla consultazione o comunque alla conoscenza di fonti manoscritte. Alquanto sorprendentemente, sembra infatti di riconoscere, nella traduzione italiana, la presenza di lezioni derivanti da l, l'edizione manoscritta del Satyricon redatta da Scaligero.

\footnotetext{
${ }^{37}$ Sulla congettura di Scaligero, vedi Iodice in Vergilius 2002, 362.
} 
Tab. 5. Confronto tra l'ed. Wouweren, Ang e l'edizione manoscritta di Scaligero (l)

\begin{tabular}{|c|c|c|c|}
\hline Sat. & ed. Wouweren & Ang & 1 \\
\hline $23,3,3$ & et manu procaces & con la mano libidinosi & [et] manu procaces \\
\hline $83,10,4$ & ad praemia peccat & gravi premi conseguiscano & ad premia spectat \\
\hline 86,7 & ergo & $\underline{i o}$ & $\underline{\text { ego }}$ \\
\hline 86,7 & amplexui & $\underline{\text { abbracciatomi }}$ & $\underline{\text { amplexu }}$ \\
\hline 92,12 & inimici nostri & del $\underline{\text { mio }}$ rivale & inimici mei \\
\hline 98,9 & $\begin{array}{l}\text { utinam Hiberum invaderet } \\
\text { mare }\end{array}$ & $\underline{\text { o }}$ mi trangugiasse il mare & $\begin{array}{l}\text { aut hibernum invaderet } \\
\text { mare }\end{array}$ \\
\hline 103,1 & inquit & $\underline{o m}$. & $\underline{o m}$. \\
\hline
\end{tabular}

E se la maggior parte delle lezioni scaligeriane ora elencate si possono leggere nelle note di Johannes Bosch in Petronius 1677, ${ }^{38}$ successivamente riprese da Burman, alcune, come mei in Sat. 92,12 o aut in Sat. 98,9, a mia conoscenza, non ricorrono in nessuna edizione precedente a Petronius 1862.

Nella traduzione italiana si intravvede forse anche la presenza di un altro insigne umanista, Marc-Antoine Muret (1526-1585). Sebbene i contemporanei attribuissero a Muret un commento sul Satyricon o addirittura un'edizione (Richardson 1993, 13-14), allo stato attuale i documenti che attestino un interesse del francese per il testo petroniano sono veramente scarni e consistono principalmente nelle note apposte da Muret alla propria copia di c (= Petronius 1520$).{ }^{39}$ In quest'ultima, in corrispondenza di Sat. 89,10, dove sono descritti i Greci che si stanno stipando nel ventre del cavallo di Troia, è chiaramente leggibile l'emendamento di pugno di Muret di et in voto latent in et in utero. Ora, in Ang si legge proprio $e$ in quell'utero racchiusi non appariscano, traduzione che sembrerebbe presupporre l'emendamento di Muret. Inoltre, anche quella che sembrerebbe essere la correzione di Muret a Sat. 89,22 di laetus resilit in latus, troverebbe parallelo nella traduzione italiana e 'l lato e 'l fianco di quel cavallo rimbombò.

Se finora abbiamo trattato di congetture e varianti che possono essere rintracciate in edizioni, commenti o annotazioni, siano essi editi o inediti, dobbiamo ora trattare di interventi emendativi che pure si deducono da Ang, ma per i quali non saprei identificare una fonte del tempo. Mi limito ad elencarne alcuni: in corrispondenza di Sat. 122,168 Ang ha Voi vincitori itene feroci, dove il testo delle

${ }^{38}$ Le Notae di Bosch sono datate 1676. Oltre a numerose altre fonti, Bosch ebbe a disposizione il manoscritto scaligeriano I (Vannini in Petronius 2010, 51).

${ }^{39}$ L'esemplare appartiene oggi alla Biblioteca Nazionale di Roma (segnatura: 69.1. F.4) che lo ha digitalizzato e reso consultabile in linea (vedi catalogo online della Biblioteca); nella sua edizione Vannini accoglie uno degli emendamenti annotati da Muret (vedi Petronius 2010, 204). 
edizioni a stampa del Satyricon sino a Petronius 1669 leggeva victores ite ferentes. La lezione furentes che leggiamo oggi e che sembra già sottintesa da Ang deriva da $\mathbf{A}$, il manoscritto di classe $\boldsymbol{\delta}$ rinvenuto contestualmente al frammento di Trau. ${ }^{40}$ Non si può tuttavia escludere che la lezione furentes possa essere scaturita per via congetturale precedentemente al 1669. Ancora, in corrispondenza di Sat. 129,6 la traduzione già mi sembri mezzo morto presume necessariamente l'espunzione medius [fidius] iam peristi, accolta da Müller in Petronius 2009 e da questi attribuita ad un contributo di Paul Thomas del 1893. Infine, la traduzione di Sat. 131,9 agitava l'aria per eccitarne il vento sottintende un'integrazione assimilabile a <aerea $>$ verberabat proposta in apparatu da Ernout in Petronius 1922 e ormai stabilmente nel testo del Satyricon.

Ma anche nella traduzione dei Fragmenta e degli Errores Venerii si riconoscono interventi che sembrano in qualche modo in anticipo sui tempi, come ad esempio in corrispondenza di $f r .36 \mathrm{~B} .=f r .32$ M. 6 dove leggiamo: appena vil panno ricuopre $i$ soldati, che presume, mi sembra, la congettura contemptus ...pannus, laddove Pithou aveva invece editato contemni ...pannus. ${ }^{41}$ Analogamente, nella traduzione del Pervigilium Veneris, in corrispondenza di PV 63, leggiamo ventre dove il testo tràdito avrebbe mentem, il che trova un interessante riscontro nella congettura ventrem per mentem proposta da Herrmann nel 1953; mentre la traduzione resta di $P V 46$ già sottende, a mio avviso, l'emendamento di detinent in detine te proposto da Orelli nel 1831 (cfr. Pervigilium Veneris 1998).

\section{Tradizione esegetica e revisori profani}

Credo risulti ormai evidente che gli interventi emendativi rivelati dalla traduzione italiana presuppongano l'azione di un autentico specialista, il quale avrebbe scremato non pochi commenti eruditi, avrebbe avuto accesso o comunque conoscenza di fonti inedite quali $\mathbf{I}$ e potrebbe da ultimo anche aver ideato alcune ottime congetture. Mi pare altrettanto evidente che in alcun modo un erudito di questo calibro possa essere intervenuto personalmente sulla traduzione italiana e il suo idiosincratico testo.

Una soluzione che mi sembra convincente è quella di ipotizzare che, se non tutti, almeno buona parte degli emendamenti riconoscibili nella traduzione fossero raccolti in un commento e che tale commento sia stato impiegato per lavorare sul

\footnotetext{
${ }^{40}$ Il codice che trasmette il frammento di Trau contiene infatti anche altri testi, tra cui per l'appunto $\mathbf{A}$. Da notare che altri due manoscritti della classe $\boldsymbol{\delta}$ presentano la lezione furentes (Richardson 1984, 100).

${ }^{41}$ L'emendamento contemptus, attribuito a Reiske, è difeso da Sommariva 2010.
} 
testo italiano. Postulo dunque l'esistenza di un commento inedito che doveva comprendere osservazioni al testo del Satyricon nonché (almeno) alla raccolta poetica degli Errores Venerii. E non credo si tratti di un'ipotesi temeraria, dal momento che sono documentati esempi di commenti manoscritti al Satyricon. Io stessa ho avuto modo di consultare le anonime In Petronii Arbitri Satyricon Notae iuxta ordinem et scripturam impressionis Parisiensis anni 1601 conservate presso la Biblioteca Vaticana, che però non hanno rivelato alcun nesso con Ang. ${ }^{42}$ È difatti tangibile la ritrosia degli eruditi ad associare pubblicamente il proprio nome a quello di Petronio, in particolare una volta venuti alla luce gli scabrosi contenuti di L. Abbiamo già avuto modo di osservare come le prime edizioni dei longiora (Petronius 1575, 1577, 1587) siano state stampate in veste anonima o come il commento di Goldast sia stato pubblicato sotto pseudonimo (vedi n.34). Altri commenti, come quello di Brassicanus, di Pierre Daniel (vedi n.29) o di Schoppe sono stati pubblicati solo a decenni di distanza dalla loro redazione. Infine, non mancano indizi concreti riguardanti delle note inedite redatte da Scaligero. ${ }^{43}$ Non reputo perciò azzardato ipotizzare che nel Seicento potesse circolare, in forma manoscritta, una silloge di note sul testo del Satyricon tratte anche da fonti a noi ignote.

Il commento inedito, di incontestabile qualità, emanerebbe dunque da ambienti umanistici e tuttavia sarebbe stato manipolato da non-specialisti. Dico questo perché nella traduzione italiana sono presenti delle sviste che a mio avviso si spiegano solo ipotizzando a monte il fraintendimento di osservazioni a carattere erudito. Una indicazione di natura critico-testuale che sembrerebbe essere stata del tutto travisata riguarda, ad esempio, l'interpretazione di [Verg.] Catal. 12,7. Il carme si prende gioco del superbo Nottuino, il quale sposando la figlia di Atilio è stato costretto a prendersi anche l'altra. Gli interpreti sono tuttora incerti nello stabilire se 'l'altra figlia di Atilio' sia il tràdito hirneam (ducit/...superbus ecce Noctuinus hirneam) o se occorra emendare in herniam. In quest'ultimo caso, il riferimento sarebbe all'ernia cui andrebbe incontro Nottuino a causa dell'eccessiva attività sessuale comportata dalle nozze; chi mantiene hirnia ('boccale di vino') ritiene invece che la figlia di Atilio sarebbe stata un'ubriacona, perciò sposando lei, Nottuino sposerebbe anche una bottiglia. ${ }^{44}$ Pithou édita hernia e così leggiamo nelle ristampe francesi dell'ed. Wouweren, su cui verosimilmente si basa la traduzione italiana. Tuttavia, questa sembra aver risentito del dibattito critico, in particolare del commento di Scaligero, il quale prendeva in considerazione

\footnotetext{
${ }^{42}$ Si tratta del ms. Barb. Lat. 51, segnalato da Rini 1937, 54 n. 332.

${ }^{43}$ Come documenta Stagni, la stessa edizione tornesiana veniva attribuita dai contemporanei a Scaligero (Stagni 1993, 224-229).

${ }^{44}$ Per una disamina più dettagliata, vedi Watson 2008.
} 
entrambe le ipotesi. A sostegno di hirnea, Scaligero scrive, ricordando un analogo epigramma greco, che la figlia di Atilio sarebbe stata dedita al vino al punto da chiamare la bottiglia 'sorella', ecco perché le figlie sarebbero due: lei e Bottiglia (ergo duae filiae, ipsa et eius soror Hirnea). E io credo che solo il totale fraintendimento di un'osservazione analoga a quella di Scaligero possa spiegare l'improbabile traduzione del verso fornita da Ang ovvero: Nottuino il superbo se ne mena a casa Hirnea.

Anche dalla traduzione di Sat. 107,15 si evince la presenza di un'indicazione del tutto travisata. Nel passo in questione Lica incalza Encolpio dicendogli: Pharmace, responde (ed. Wouweren). Il grecismo pharmacus rappresenta un hapax nella lingua latina per il quale diverse interpretazioni sono state avanzate dai critici (vedi Vannini in Petronius 2010, 192; Schmeling 2011, 419). La traduzione italiana tiene certamente presente l'interpretazione risalente a Dousa, ripresa in molti commenti, secondo la quale pharmacus vorrebbe dire 'scellerato, empio', ma è altrettanto evidente che l'indicazione è stata erroneamente compresa, poiché in Ang leggiamo: Rispondi, scelerato più di Farmaco!. Parimenti, solo il travisamento di una nota di commento può spiegare lo straniante le prugne strepitose ('crepitanti') si fanno sentire su la fiamma, che traduce fr.45 B. = fr. 48 M. 6: tepido consonat igne rogus ('un rogo ardente scoppietta col suo tiepido fuoco', trad. di Sommariva 2004, 97). Quello che possiamo supporre è che nel commento venisse delucidata l'espressione tepido igne, specificando che il fuoco tiepido e scoppiettante è quello dei tizzoni ardenti (pruna, prunae) e che questo termine possa essere stato equivocato con prunum, pruni 'prugna'.

Ma la prova più schiacciante del fatto che la traduzione sia stata rivista da un profano sulla scorta di un commento dotto è rappresentato dalla saltuarietà degli interventi operati su TR1, in cui si trovano ottime correzioni incastonate nel testo obsoleto derivato dalla fonte pre-tornesiana. Questa evidenza porta infatti a concludere che a TR1 sia stato sovrapposto un commento concepito in epoca posttornesiana, quando ormai, comprensibilmente, il testo delle prime edizioni a stampa del Satyricon, una volta venuti alla luce gli excerpta longiora, non suscitava più alcun interesse. Con l'eccezione dell'onnicomprensivo commento di Burman, nessuno dei commenti finora elencati si preoccupa di segnalare che in Sat. 94,2 Circlopius sia da correggere in Encolpius o che in Sat. 88,5 occorra integrare Lysippum (vedi tab. 1). È dunque verosimile che anche il commento a disposizione di chi ha riletto TR1 sorvolasse del tutto sulle lezioni patentemente erronee delle edizioni pre-tornesiane. Dobbiamo quindi concludere che chi ha emendato la traduzione italiana è intervenuto non su iniziativa personale ma attenendosi pedissequamente ad uno strumento esegetico che doveva avere a disposizione. 
Se la traccia di un commento maldestramente maneggiato si coglie con evidenza nel testo italiano, resta problematico da determinare quando la traduzione sarebbe stata sottoposta al processo di revisione: è stato il traduttore stesso a giovarsi del commento o dobbiamo pensare ad un successivo revisore? La distanza temporale che sussiste fra la presunta data di composizione di TR e la pubblicazione dell'ed. Gonzáles fa decisamente propendere per questa seconda ipotesi. Peraltro, Ang presenta delle interpolazioni che suggeriscono che la traduzione sia stata sottoposta, successivamente alla sua composizione, ad almeno una rilettura. Mi sono già soffermata sulla presenza, in Ang, di glosse che commentano il Satyricon fornendo informazioni a carattere erudito. Oltre a queste, si riscontrano anche alcune interpolazioni che visibilmente conservano l'eco di una riflessione critica sul testo del Satyricon. Si consideri l'esempio che segue: il dotto Epicuro... disse che questo è il fine della nostra vita, telos (i. il fine) (traduzione di Sat. 132,15,7-8). Apparentemente la glossa spiega il significato di telos ('il fine'), ma a ben vedere essa è totalmente inutile, dal momento che la traduzione già sottintendeva la lezione telos dell'ed. Wouweren; non solo, ma per un lettore l'inserimento di un termine come telos non semplifica ma, tutto al contrario, complica il testo. Sorge quindi il sospetto che il copista abbia interpolato nel testo quella che doveva essere una semplice annotazione desunta da una fonte erudita e riportata a margine della traduzione. ${ }^{45}$

Analogamente, in corrispondenza di Sat. 119,11, in Ang si legge: dall'Africa $i$ marmi (i. croste, stipiti delle porte). Si tratta di un verso dove Müller in Petronius 2009 lascia una crux: hinc Numidae $†$ accusatius $\uparrow$. Ma già nelle Animadversiones di Wouweren si può trovare (anonima) la proposta di Scaligero di leggere hinc Numidae crustas, dove crustas è da intendere nel senso di 'rivestimenti di marmo'. Come si sarà notato, la glossa di Ang in realtà non spiega affatto il testo italiano, di per sé trasparente, ma traduce la congettura di Scaligero, peraltro malamente. Anche qui si ha l'impressione che un'annotazione estemporanea sia stata inglobata nel testo senza discernimento alcuno.

Non sono inoltre rari in Ang casi di accumulo di lezioni alternative, che reputerei derivare anche esse dall' incorporamento di marginalia nel testo continuo. Un esempio è dato dalla traduzione di [Verg.] Catal. 13,7 (dagli Errores Venerii), dove si legge Che più ti duole, o i fianchi, o gli scherzi? in cui si rileva la coesistenza del tràdito an ioci dolent? ('forse gli scherzi ti fanno male?) e della congettura di Dousa (an loci dolent?), secondo il quale a fare male sarebbero i

${ }^{45}$ L'intero passo in Ang legge: il dotto Epicuro... disse che questo è il fine della nostra vita, telos (i. il fine). Ede, bibe, lude, post mortem nulla voluptas. Mi riservo di commentare in un altro contributo la presenza del motto pseudo - epicureo. 
lombi. ${ }^{46}$ Oppure, nella traduzione del Satyricon, si possono trovare affiancate lezione pre-tornesiana e lezione $\mathbf{L}$, a partire dall'incipit (Sat. 1,1): Forsi con altra sorte di furie, dove convivono il num di $\mathbf{L}$ e il cum di $\boldsymbol{\delta}$ o, anche in corrispondenza di Sat. 111,12: pensi tu che le ceneri de' morti o sentano o si curano delle tue angosce?, traduzione che presenta al contempo la lezione sentire di $\boldsymbol{\delta}$ e la lezione curare di L. Così in Sat. 126,12, la glossa continuando ...il nostro burlare (il nostro parlare) propone la lezione iocis ('gli scherzi, il burlare'), propagatasi a partire dall'ed. Goldast, in concomitanza con procedentibus...logis ('i discorsi, il parlare') della tradizione precedente. In corrispondenza di Sat. 113,11 troviamo poi un esempio particolarmente interessante. Qui Ang legge: tanto conto farai di costei, quanto d'una vile sporta (i. puttana). Come ovvio, in italiano sporta non vuol dire 'puttana': la glossa rinvia in realtà alla proposta di Elias Putsch (15801606), comunemente accettata dagli editori di Petronio, di emendare il tràdito sportam con scortum. Abbiamo quindi una variante, che in questo caso non solo è stata interpolata nella traduzione, ma è anche stata erroneamente trattata dal copista alla stregua di una glossa esplicativa.

\section{Conclusioni}

Da quanto appena esposto risulta quanto possa essere problematico tentare di stabilire l'esatta successione degli interventi operati sulla traduzione, nel cui testo si sovrappongono più fonti e più mani, il tutto mascherato dall'operato del copista. Tentando di ridurre al minimo il numero degli attori, dobbiamo comunque prendere in considerazione, oltre al traduttore e al copista, almeno un revisore, che a distanza di qualche decennio avrebbe riletto e annotato la traduzione sulla base di fonti erudite. E, volendo attenerci ad un principio di stretta economia, attribuiremo allo stesso anche le glosse che commentano l'italiano della traduzione con una varietà più moderna. Comunque, al di là dell'esatta quantificazione delle mani intervenute sul testo, quello che più mi interessa sottolineare è come ogni aspetto di Ang testimoni della circolazione della traduzione tra lettori che di certo possiamo definire non eruditi o anche popolari, intendendo con questo termine ' $\mathrm{i}$ lettori più umili (artigiani, bottegai, piccoli commercianti, élites paesane)' (Chartier 1995, 321).

Anzi, io credo che la traduzione sia stata proprio prodotta per simile lettorato. Prova ne è la qualità stessa della traduzione, iniziata sugli excerpta brevia e terminata con i longiora, il che fa pensare ad un lavoro condotto in tutta fretta e per

${ }^{46}$ La congettura di Dousa e relativa spiegazione, si può leggere in Vergilius 1963. 
un pubblico poco esigente. Le successive revisioni, oltre a provare l'effettiva circolazione della traduzione, ne confermano la destinazione popolare, in particolare per la presenza delle glosse che esplicitano, ricorrendo al dialetto, l'italiano del traduttore. Anche dall'interferenza del dialetto nel lavoro del copista si evince una derivazione non certo colta, per finire con la materialità di Ang e quell'appunto vergato sulla coperta (farina orzo fave), che lascia veramente immaginare il libro nella bottega di un commerciante.

Constatare la diffusione del Satyricon in un simile contesto socio-culturale certo sorprende, perché la fortuna cinque-seicentesca di Petronio è stata finora documentata esclusivamente in relazione al mondo erudito. L'interrogativo che si pone direi prepotentemente è quindi: come spiegare la presenza di Petronio presso un lettorato popolare? Certamente, i contenuti proibiti del Satyricon possono aver costituito motivo di attrazione, tuttavia non ritengo che la sola presenza di passi pornografici sia sufficiente a giustificare la diffusione di Petronio tra lettori non eruditi. Questo per un fatto abbastanza ovvio, ovvero che nella coeva letteratura italiana non mancano certo testi erotici e non si vede dunque la necessità di andare a tradurre (e con tanta difficoltà) un testo ostico, frammentario, come il Satyricon se lo scopo fosse semplicemente quello di fornire una lettura licenziosa.

Piuttosto, penso che il motivo del successo vada ricercato nella specificità del Satyricon di rappresentare (presumibilmente) l'inversione parodica del romanzo greco. ${ }^{47}$ Se infatti molti dei rimandi intertestuali che intessono il Satyricon sono destinati a rimanere preclusi ad un lettore che non sia più che avvertito, non è affatto azzardato ritenere che anche un ordinario lettore del Seicento fosse in grado di cogliere il sovvertimento del genere romanzesco operato da Petronio, tanto più evidente nel Satyricon privo dell'episodio della Cena Trimalchionis. E questo non solo perché i romanzi greci (in particolare le Etiopiche di Eliodoro) ebbero larga diffusione al tempo, come attestano le traduzioni e gli adattamenti in tutta Europa ${ }^{48}$ ma soprattutto perché, come sottolineato da Cavaillé, l'amore, così come idealizzato nel romanzo greco, si era ormai costituito come un vero e proprio linguaggio comune dell'Occidente, teorizzato dalla trattatistica neoplatonica, assorbito dalla morale cristiana e decisamente incombente nella letteratura italiana (Cavaillé 2013, 33).

${ }^{47}$ Sul dibattuto rapporto del Satyricon con il romanzo greco, vedi Conte 1996, 29-35. Di particolare interesse l'analisi di Setaioli 2009.

${ }^{48}$ Per la Francia e la Gran Bretagna, vedi Sandy 1996. Non ho trovato studi specifici sull'Italia, ma già i dati bibliografici sono fortemente indicativi del successo del volgarizzamento delle Etiopiche ad opera di Leonardo Ghini (1535-1589) pubblicato nel 1556 e ristampato ben 9 volte tra Cinque e Seicento, vedi la sezione 'Italian translations' in Ricquier 2018, 1-34, in questo stesso numero di Ancient Narrative. 
Parte della critica petroniana è incline ad interpretare la parodia di Petronio nei confronti del romanzo greco come la reazione di un raffinato letterato nei confronti di un trito modello letterario. Non si può tuttavia negare che il Satyricon potesse prestarsi ad essere letto, in piena Controriforma, come dissacrante sovvertimento non tanto del genere romanzesco quanto dei valori (castità, fedeltà, pietà religiosa) da questo veicolati (cfr. Conte 1996, 104-139). Oltre tutto, anche i componimenti raccolti negli Errores Venerii si prestano ad un'analoga interpretazione, dal momento che in prevalenza trattano di rapporti amorosi piuttosto distanti dal petrarchismo. E questa ricezione 'ideologica' del Satyricon che prospetto non rappresenterebbe neanche un unicum, poiché nel Seicento italiano proliferano gli scritti che rovesciano la visione idealizzata dell'amore e della donna, per di più spesso proprio attraverso la provocatoria esaltazione della sodomia (Cavaillé 2013,36). Mi chiedo anzi se questa interpretazione non possa spiegare più in generale la progressiva diffusione, nel corso del Seicento, del Satyricon al di fuori della cerchia ristretta degli umanisti, testimoniata dal debordante successo editoriale di Petronio. ${ }^{49}$

\section{Appendice}

Si ritiene comunemente che la prima ed. Wouweren sia stata pubblicata nel 1594 a Leida (cfr. Schmeling e Stuckey 1977, 52). Trovo la prima menzione di un'edizione del Satyricon risalente al 1594 nella bibliografia dell'ed. Bipontina, che riporta piuttosto succintamente: 1594 Lugduni Bat. 12 T. Petronii Arbitri Satyricon (Notitia Literaria in Petronius 1790, xxiv). Tuttavia, un'edizione di Petronio risalente al 1594 risulta di fatto introvabile. Quello che sospetto è che la presunta edizione del 1594 altro non sia se non l'ed. Wouweren del 1614 erroneamente catalogata.

L'errata interpretazione dell'anno di pubblicazione, con la confusione tra 1594 e 1614, potrebbe essere stata generata da molteplici fattori. Anzitutto, dal fatto che sul frontespizio dell'ed. del 1614 l'anno di pubblicazione è scritto con la notazione in voga nei Paesi Bassi, ovvero con le cifre romane invertite e perciò

\footnotetext{
${ }^{49}$ Questo articolo è stato elaborato nell'ambito del progetto di ricerca Popular readers and clandestine literature: the case of an early modern translation of Petronius' Satyricon into Italian (17th C.) (ID 707253 Programma Horizon 2020) interamente finanziato dalla Commissione Europea mediante la borsa di post-dottorato Marie Skłodowska-Curie Career Restart Panel assegnatami. Ringrazio il mio supervisore Jean-Pierre Cavaillé e il Grihl, per avermi dato la possibilità di presentare e discutere il mio lavoro nel corso del seminario 2017-2018 'Écritures du passé: histoire et littérature'.
} 
facilmente equivocabile altrove (vedi Schmeling e Stuckey 1977, 48 imm. 4). Si noti a tal proposito che l'unico esemplare dell'ed. Wouweren del 1594 recensito da Schmeling e Stuckey, posseduto dalla Bayerische StaatsBibliothek di Monaco di Baviera, si è rivelato poi essere per l'appunto una copia dell'ed. 1614. Per parte mia posso segnalare un problema analogo con la copia dell'ed. del 1614 posseduta dalla Biblioteca del Monumento Nazionale di Trisulti (segnatura: INN. 2 A 168/2) catalogata come édita nel 1514, nonché con la copia posseduta dalla Bibliothèque Interuniversitaire de la Sorbonne di Parigi (segnatura: R.ra 96 Nains) sul cui frontespizio una mano aveva convertito la data in cifre arabe come '1594'. La nota è stata successivamente biffata e l'esemplare è oggi correttamente catalogato come Petronius 1614.

Ad alimentare la confusione può anche avere contribuito il fatto che l'ed. del 1614 non include la lettera dedicatoria di Wouweren a Scaligero datata 1595 che apre l'ed. Wouweren del 1596 e si trova nella maggior parte delle ripubblicazioni dell'ed. Wouweren. Si aggiunga inoltre che il frontespizio dell'ed. del 1596 presenta la pubblicazione come Nova Editio, il che potrebbe in effetti portare a pensare che si tratti di una nuova ed. Wouweren - con il sottinteso che altre siano già state pubblicate in precedenza. Ma, come sottolinea Stagni, i tipografi al tempo erano soliti presentare le stampe dei classici con formule altisonanti quanto mendaci (amplius, ex veteribus libris emendatius et amplius, accedunt numquam antehac exclusa, ...), atte a sottolineare la novità del prodotto (Stagni 1993, 227 n.50). Si consideri infine che nella biografia di Wouweren non si trova alcuna menzione di un'edizione pubblicata dal tedesco nel 1594 (vedi Deitz 1995, 138).

Per i motivi appena esposti, sono propensa a ritenere che l'ed. Wouweren del 1594 non sia mai esistita. Altrettanto direi dell'ed. Wouweren del 1595 indicizzata da Schmeling e Stuckey 1977, 52. L'unica copia di questa edizione risulterebbe quella posseduta dalla Bayerische StaatsBibliothek di Monaco di Baviera. La copia è consultabile on line (segnatura: A. lat.1388) e risulta mutila del frontespizio. Sull'ultima carta di guardia prima del testo stampato, si legge annotato a matita 1595, datazione che ritengo essere stata dedotta, in assenza del frontespizio, dalla lettera dedicatoria a Scaligero. Occorre inoltre prestare attenzione al fatto che l'esemplare in questione presenta un commento intitolato Symbolae Annotationum nel quale sono più praticamente fatti confluire i sei commenti pubblicati separatamente nell'ed. Wouweren del 1596, come peraltro spiega il tipografo stesso al lettore introducendo tale commento. La prima ed. Wouweren a presentare questa soluzione è l'ed. del 1604, soluzione successivamente ripresa in Petronius 1623, 1626, e 1634. Ora, il formato e la composizione tipografica dell'esemplare catalogato dalla Bayerische StaatsBibliothek come édito nel 1595 coincidono 
esattamente con quelli di Petronius 1634. Ritengo perciò che l'esemplare posseduto dalla Bayerische StaatsBibliothek altro non sia se non una copia di Petronius 1634 erroneamente datata.

\section{Bibliografia}

Barbierato, F. 2012. The Inquisitor in the Hat Shop: Inquisition, Forbidden Books and Unbelief in Early Modern Venice, Farnham: Ashgate.

Carnelos, L. 2012. 'Con libri alla mano': l'editoria di larga diffusione a Venezia tra Sei e Settecento, Milano: Unicopli.

Cavaillé, J.-P. 2013. Les Déniaisés. Irréligion et libertinage au début de l'époque moderne, Parigi: Classiques Garnier.

Chartier, R. 1995. 'Letture e lettori «popolari» dal Rinascimento al Settecento', in: G. Cavallo e Id. (a c. di), Storia della lettura nel mondo occidentale, Roma - Bari: Laterza, 317-335.

Cointre, A. 2014. 'Prose narrative', in : Y. Chevrel, A. Cointre e Y.-M. Tran-Gervat (dir.), Histoire des traductions en langue française: XVIIe et XVIIIe siècles, Parigi: Verdier, 11211209.

Conte, G.-B. 1996. The Hidden Author. An Interpretation of Petronius' Satyricon, BerkeleyLos Angeles-Londra: University of California Press.

Deitz, L. 1995. 'Ioannes Wower of Hamburg, Philologist and Polymath. A Preliminary Sketch of His Life and Works', JWI 58, 132-151.

De la Mare, A. 1976. 'The Return of Petronius to Italy', in: J.J. G. Alexander e M.T. Gibson (a c. di), Medieval Learning and Literature. Essays presented to Richard William Hunt, Oxford: Clarendon, 220-251.

Grafton, A. 1993. Joseph Scaliger: A study in the History of Classical Scholarship. 2: Historical Chronology, Oxford: Clarendon.

Laes, C. 1988. 'Forgering Petronius: François Nodot and the fake Petronius Fragments', HumLov 47, 358-402.

Mattesini, E. 1992. 'L’Umbria', in: F. Bruni (dir.), L'italiano nelle regioni. Lingua e identità nazionale, Torino: UTET, 507-539.

Onelli, C. 2012. 'Con oscurità mutando in nomi: Napoli epicurea nei Successi di Eumolpione (1678)', California Italian Studies 3, $1<$ https://escholarship.org/uc/item/2tr7x1nd $>$.

Pellegrini, G. B. 1982. 'Rassegna di studi dialettologici umbri', Studi Mediolatini e Volgari 2930, 183-204.

Pervigilium Veneris 1998. Pervigilium Veneris, introduzione, testo critico, traduzione e commento di Crescenzo Formicola, Napoli: Loffredo.

Petronius 1520. Petronii Arbitri quatenus extare comperitur Satyrae Fragmentum..., Parigi: Regnault Chaudière.

- 1565. Petronii Arbitri Massiliensis Satyrici Fragmenta restituta et aucta e bibliotheca Iohannis Sambuci, Anversa: Christophe Plantin.

- 1575. Petronii Arbitri Satyricon, Lione: Jean de Tournes.

- 1577. Petronii Arbitri Satyricon ex veteribus libris emendatius et amplius, Parigi: Mamert Patisson.

- 1587. Petronii Arbitri Satyricon. Adiecta sunt veterum quorundam poetarum carmina non dissimili argumenti..., Parigi: Mamert Patisson. 
- 1596. Petronii Arbitri Satyricon cum notis et observationibus variorum, Leida: François Raphelenge.

- 1601. Petronii Arbitri Satyricon cum notis et observationibus variorum, Parigi (10 emissioni).

- 1604. Petronii Arbitri Satyricon cum uberioribus commentarii instar notis..., Leida: François Raphelenge.

- 1608. Petronii Arbitri Satyricon. Multis virorum illustrium notis et observationibus illustratum, Lione: Paul Frellon.

- 1610. T. Petronii Arbitri Equitis Romani Satiricon..., [Francoforte]: Johann Bringer.

- 1614. Petronii Arbitri Satyricon, Leida: François Raphelenge.

- 1615. T. Petronii Arbitri Equitis Romani Satyricon...Noviter recensitum, interpolatum et auctum, Lione: Paul Frellon.

- 1618a. Petronii Arbitri Equitis Romani Satyricon...Noviter recensitum, interpolatum et auctum, Lione: Paul Frellon.

- 1618b. T. Petronii Arbitri Satyricon, Leida: Jacob Marcus.

- 1623. Petronii Arbitri Satyricon, Leida: Jan Maire.

- 1626. Petronii Arbitri Satyricon..., Amsterdam: [Willem Blaeu].

- 1629a. T. Petronii Arbitri Equitis Romani Satyricon..., [Ginevra]: Jean Mercier.

- 1629b. T. Petroni [sic] Arbitri E. R. Satiricon. Extrema editio ex musaeo D. Iosephi Antoni [sic] Gonsali de Salas..., Francoforte: Wolfgang Hofmann.

- 1629c. T. Petroni [sic] Arbitri Satyricon ....Noviter Recensente Jo. Petro Lotichio..., Francoforte: Lukas Jennis.

- 1634. Petronii Arbitri Satyricon..., Amsterdam: Jan Jansson.

- 1669. Titi Petronii Arbitri Equitis Romani Satyricon cum fragmento nuper Tragurii reperto..., concinnante Michaele Hadrianide, Amsterdam: Jan Blaeu.

- 1709. Titi Petronii Arbitri Satyricon quae supersunt...curante Petro Burmanno, Utrecht: Willem van de Water.

- 1790. T. Petronii Arbitri Equitis Romani Satiricon...Praemittitur notitia literaria studiis Societatis Bipontinae..., Zweibrücken: Societas Bipontina.

- 1862. Petronii Arbitri Satirarum reliquiae ex recensione Francisci Buecheleri, Berlino: Weidmann.

- 1863. The manuscripts of the Satyricon of Petronius Arbiter, described and collected by Charles Beck, Cambridge (Mass.): Riverside Press.

- 1922. Le Satiricon, texte établi et traduit par Alfred Ernout, Parigi: Les Belles Lettres.

- 1995. Satyricon, introduzione, traduzione e note di Andrea Aragosti, Milano: Biblioteca Universale Rizzoli.

- 2009. Petronii Arbitri Satyricon Reliquiae, edidit K. Müller, editio iterata correctior editionis quartae (1995), Berlino-New York: De Gruyter.

- 2010. Petronii Arbitri Satyricon 100-115. Edizione critica e commento di Giulio Vannini, Berlino-New York: De Gruyter.

Richardson, W. 1984. 'A New Renaissance Petronius Manuscript: Indiana, Notre Dame 58', Scriptorium 38, 1, 89-100.

- 1993. Reading and Variant in Petronius: Studies on the French Humanists and Their Manuscript Sources, Toronto- Buffalo-Londra: University of Toronto Press.

Ricquier, K. 2018. 'The early modern transmission of the ancient Greek romance: a bibliographic survey', $A N$ 15, 1-34.

Riffaud, A. 2011. Une archéologie du livre français moderne, Ginevra: Droz. 
Rini, A. 1937. Petronius in Italy: From the Thirteenth Century to the Present Time, New York: Cappabianca Press.

Sandy, G. 1996. 'The Heritage of the Ancient Greek novel in France and Britain', in: G. Schmeling (a c. di), The Novel in the Ancient World, Leida - New York - Köln: Brill, 736773.

Scaliger, J.-J. 2012. The Correspondance of Joseph Justus Scaliger. Vol. 2., a c. di P. Botley e D. van Miert, Ginevra: Droz.

Schmeling, G. 2011. A Commentary on the Satyrica of Petronius, Oxford: Oxford University Press.

Schmeling, G. e Stuckey, J. H. 1977. A Bibliography of Petronius, Leida: Brill.

Sciarra, E. 2009. 'Breve storia del fondo manoscritto della biblioteca Angelica', La Bibliofilia $111,3,251-281$.

Serianni, L. 1990. Storia della Lingua Italiana: il secondo Ottocento: Dall'Unità alla prima guerra mondiale, Bologna: Il Mulino.

Setaioli, A. 2009. 'L'amour romanesque entre idéal et parodie: les romanciers grecs et Pétrone', Rursus $4<$ http://journals.openedition.org/rursus/295>.

Sommariva, G. 2004. Petronio nell'«Anthologia Latina». Parte prima: I carmi parodici della poesia didascalica, Sarzana: Agorà.

— 2010. Petronio nell' «Anthologia Latina». Parte seconda: I carmi su temi diatribici ed etici, Lugano: Athenaion.

Stagni, E. 1993. 'Ricerche sulla tradizione manoscritta di Petronio: l'edito princeps dei 'longa' e i codici di Tornesio', MD 30, 205-230.

Vergilius 1573. Publii Virgilii Maronis Appendix...Iosephi Scaligeri in eandem Appendicem commentarii et castigationes..., Lione: Guillaume Rouillé.

- 1963. Appendix Vergiliana. Testo, traduzione e note critiche di Armando Salvatore, Napoli: Libreria scientifica editrice.

- 2002. Appendix Vergiliana. Prefazione di Luca Canali, a cura di Maria Grazia Iodice, Milano: Mondadori.

Vitale, S. 1934. Roma. R. Biblioteca Angelica, in A. Sorbelli (dir.), Inventari dei manoscritti delle Biblioteche d'Italia. Opera fondata da G. Mazzatinti, vol. 56, Firenze: Olschki.

Watson, L. C. 2008. 'Of Hernias and Wine-Jugs: Catalepton 12', Mnemosyne 61, 245-256. 